\title{
Aufsatz
}

Thomas Freller

\section{Nach Lepanto: Osmanische Strategie und Seekriegspraxis im Mittelmeer aus der Perspektive eines deutschen Reisenden}

DOI 10.1515/mgzs-2015-0005

Zusammenfassung: Das Tagebuch des Königsberger Apothekers Reinhold Lubenau ist eines der sehr seltenen Beispiele von authentischen Aufzeichnungen eines an einer osmanischen Inspektions- und Kaperfahrt teilnehmenden christlichen Augenzeugen. Die sich mit Themen frühneuzeitlicher türkischer Kultur, Kunst, Folklore und Politik beschäftigende Forschung hat Lubenaus Aufzeichnungen über seinen Aufenthalt in Istanbul 1587/88 bereits ausgewertet, die folgenden Notizen über seine Teilnahme an Hassan Paschas Unternehmung wurden von Studien, die sich mit der Kriegführung im Mittelmeer befassen, bisher jedoch nicht zur Kenntnis genommen. Dabei enthalten diese Aufzeichnungen - neben direkten Einblicken in die Seekriegspraxis - wertvolle Informationen über militärische Planung und Operationen der Osmanen, über Kanäle der Information und Einholung militärischer Nachrichten, sowie über die strategische Integration der nordafrikanischen Satellitenreiche Tunis, Tripolis und Algier im Kontext des Versuchs, über den östlichen und zentralen Mittelmeerraum die Kontrolle auszuüben und umfangreiche Plünderungszüge durchzuführen. Wie Lubenaus Notizen andeuten, markierte die Niederlage von Lepanto nicht das Ende osmanischer Bestrebungen, militärisch im westlichen Mittelmeer vorzugehen und die dortigen Küstenregionen $\mathrm{zu}$ bedrohen.

Schlüsselwörter: Osmanisches Reich, Mittelmeer, 16. Jahrhundert, Marinegeschichte 


\section{Reinhold Lubenau und das Osmanische Reich in der zweiten Hälfte des 16. Jahrhunderts}

Die Niederlage der Osmanen vor der Johanniterresidenz Malta im Sommer $1565^{1}$ und die Seeschlacht von Lepanto mit dem Sieg der »Heiligen Liga« am 7. Oktober 1571 gelten gemeinhin als Wendepunkte der Geschichte der osmanischen maritimen Expansion und als Ende der islamischen Bedrohung des westlichen Mittelmeerraums ${ }^{2}$. Der vorliegende Beitrag soll prüfen, inwiefern diese Feststellung einem modernen, eher durch Sekundärliteratur und verallgemeinernde Darstellungen geschuldeten Blickwinkel entstammt und der zeitgenössischen »Alltagsgeschichte und Wahrnehmung nicht gerecht wird.

Gerade die damals intensivierte Einbeziehung der nordafrikanischen Fürstentümer Tripolis, Tunis und Algier in die osmanische Hemisphäre ermöglichte der Marine der Osmanen auch nach der Niederlage von Lepanto ein wirkungsvolles und nachhaltiges Operieren im westlichen Mittelmeerraum. Diese sehr flexibel durchgeführten Operationen führten zu keinen größeren Seeschlachten tatsächlich etikettiert die Geschichtsschreibung die Jahre zwischen 1580 und 1700 als Epoche des Korsarenkrieges im Mittelmeer - und verleitete vielleicht aus diesem Grund, die weiterhin bestehende Schlagkraft der osmanischen Marine zu unterschätzen. Diese Operationen bildeten - wie im Folgenden zu zeigen sein wird - nicht nur aufgrund der zur Verfügung stehenden nordafrikanischen Basen, sondern auch aufgrund des engen Verhältnisses mit Frankreich für die katholischen Anrainerstaaten ein hohes Gefahrenpotenzial.

Das Phänomen der »Türkenfurcht« (»Turcophobia«) blieb bis in die zweite Hälfte des 17. Jahrhunderts sowohl an den italienischen Städten des Tyrrhenischen Meeres, auf den Balearen als auch entlang der spanischen Levante ein beherrschender - und realer - Faktor für Gesellschaft und Wirtschaft, der auch in der Kunst und im kollektiven Gedächtnis der »Alltagskultur« und einer steten

1 Vgl. die Behandlung des Themas bei Ernle Bradford, The Great Siege, London 1961; From the Great Siege to the Battle of Lepanto. Ed. by George Cassar, Malta 2011; Stephen C. Spiteri, The Great Siege: Knights vs Turks, Malta 2005. Der osmanische Standpunkt wird untersucht in The 1565 Ottoman Malta Campaign Register. Ed. by Arnold Cassola, Malta 1998.

2 Diese Thesen werden unter anderem vertreten von Roger Crowley, Empires of the Sea. The Siege of Malta, the Battle of Lepanto, and the Contest for the Center of the World, New York 2009; Niccolò Capponi, Victory of the West. The Story of the Battle of Lepanto, London 2006; B. Rogerson, The Last Crusaders. The Hundred-year Battle for the Centre of the World, London 2009; Hugh Bicheno, Crescent and Cross. The Battle of Lepanto 1571, London 2003. Vgl. auch die ältere Abhandlung von Wolfram zu Mondfeld, Der sinkende Halbmond. Die Seeschlacht von Lepanto im Jahre 1571. Vorbereitungen, Schlachtgeschehen, Auswirkung, Würzburg 1973. 
und teuren Verteidigungsbereitschaft ihren Niederschlag fand ${ }^{3}$. Besonders im Rahmen des in den letzten Jahrzehnten etablierten Forschungsbereichs der »Mediterranean Studies« werden diese regionen-, kultur- und konfessionsübergreifenden Themen neu und häufig interdisziplinär interpretiert, Hintergründe für Paradigmenwechsel in der okzidentalen und orientalen Politik und politische, wirtschaftliche, demografische und kulturelle Wechselwirkungen im Orbit des »Meers zwischen den Ländern ${ }^{4}$ klarer und umfassender herausgearbeitet. Die Edition von Fachzeitschriften wie »Journal of Mediterranean Studies « ${ }^{5}$ oder »Library of Mediterranean History « ${ }^{6}$ hilft hier substanziell zum besseren Verständnis des Mittelmeers als gemeinsamen kulturgeografischen Raum mit allen seinen Facetten beizutragen ${ }^{7}$.

Wie kaum eine andere authentische zeitgenössische Quelle eröffnet das bisher nur von der Turkologie und Kulturwissenschaft genutzte Tagebuch des Königsberger Apothekers Reinhold Lubenau Einblicke in die marine- und militärgeschichtlichen Aspekte dieser Situationen im Kräftefeld zwischen Orient und Okzident. Mit Lubenaus Tagebuch eröffnet sich uns - die synthetisierenden, verallgemeinernden Darstellungen der Lage verlassend - die Welt des maritimen und militärischen Alltags.

Es gibt insgesamt nur sehr wenige authentische Aufzeichnungen abendländischer Augenzeugen, die aus gleichsam interner Perspektive über osmanische maritime Unternehmungen gegen christliche Ziele berichten. $\mathrm{Zu}$ nennen wären

3 Vgl. Alexander H. de Groot, The Ottoman Threat to Europe, 1571-1800: Historical Fact or Fancy? In: Hospitaller Malta 1530-1798. Studies on Early Modern Malta and the Order of St John of Jerusalem. Ed. by Victor Mallia-Milanes, Malta 1993, S. 253f.; Charles Dalli, In Fronteria Barbarorum: Waiting for the Turks on late medieval Malta. In: Proceedings of History Week 1994, Malta 1996, S. 115-126; Carl Göllner, Turcica. Die europäischen Türkendrucke des XVI. Jahrhunderts, 3 Bde, Bukureşti [u.a.] 1961-1978. Speziell zum spanischen Blickwinkel vgl. Miguel Angel de Bunes Ibarra, La Imagen de los Musulmanes y del Norte de Africa en la España de los Siglos XVI y XVII. Los Caracteres de una Hostilidad, Madrid 1989; Andrew C. Hess, The forgotten Frontier. A History of the Sixteenth-Century Ibero-African Frontier, Chicago, London 1974, S. 84 f.

4 Hier zitiert nach David Abulafia, Das Mittelmeer. Eine Biographie, Frankfurt a.M. 2013, S. 17.

5 Das Journal of Mediterranean Studies wird seit 1990 vom Mediterranean Institute der University of Malta herausgegeben.

6 Die Gründung von Library of Mediterranean History erfolgte an der University of Malta im Jahr 1994 unter Federführung des damaligen Leiters Professor Victor Mallia-Milanes. Im selben Jahr erschien auch der erste Band dieser Fachzeitschrift.

7 Vgl. auch die kürzlich erschienenen Synthesen von David Abulafia, The Great Sea. A Human History of the Mediterranean, Oxford 2011; Construiere la Méditerranée, penser les transferts cultures. Appoches historiographiques et perspectives de recherché. Ed. par Rania Abdellatif [u.a.], München 2012; Salvatore Bono, Piratentum und Korsaren im Mittelmeer. Seekrieg, Handel und Sklaverei vom 16. bis 19. Jahrhundert, Stuttgart 2009. 
hier der über zwei Jahrzehnte nach seiner Freilassung aus osmanischer Gefangenschaft niedergeschriebene Bericht des Pfälzers Michael Heberer (»Aegyptiaca Servitus « $)^{8}$, die Notizen des Schwaben Hans Ulrich Krafft ${ }^{9}$ oder die »Neue Reysbeschreibung eines Gefangenen Christen « des Franken Johann Wild ${ }^{10}$. Mit Ausnahme Heberers berichten die übrigen, zeitweise auf Schiffen der Osmanen Sklavendienste leistenden Christen jedoch kaum Interna über den Zustand, die Einsatzweise und personelle Ausstattung osmanischer Kriegsschiffe sowie über ihre Rolle im strategischen Konzept der Osmanen.

Der erstmals 1610 gedruckte Bericht Michael Heberers erfuhr in den letzten Jahrzehnten von verschiedenster Seite wissenschaftliche Aufmerksamkeit ${ }^{11}$. Dagegen erfuhren die unmittelbar zeitgleich entstandenen, bis in das 20. Jahrhundert unpubliziert gebliebenen Aufzeichnungen des Königsberger Apothekers Reinhold Lubenau ${ }^{12}$, hinsichtlich ihrer wertvollen Qualitäten als Quelle marineund militärhistorischer Details bisher kaum Aufmerksamkeit. Im Vergleich zu den erst viele Jahre nach den Geschehnissen publizierten und redaktionell überarbeiteten Publikationen Heberers oder Wilds handelt es sich im Fall Lubenaus um unmittelbar während bzw. unwesentlich danach erstellte, strikt autoptische Aufzeichnungen. Nachträglich vorgenommene Interpolationen scheinen in der von Wilhelm Sahm 1914 und 1915 edierten Fassung nicht vorgenommen worden zu sein. Der Vergleich mit anderen zeitgenössischen Quellen und Archivalien bestätigte bisher Lubenaus Angaben in allen Aspekten.

Es kann im begrenzten Rahmen dieses Beitrags nicht Aufgabe sein, den durchaus interessanten Lebensweg des Königsberger Apothekers und späteren Ratsherren detailliert nachzuzeichnen. Gemäß seinen eigenen Angaben wurde er am 5. August 1556 als Sohn des Brauers Martin Lubenau geboren ${ }^{13}$. Nach der

8 Michael Heberer, Aegyptiaca Servitus, Heidelberg 1610. Heberer hielt sich als türkischer Galeerensklave zwischen 1585 und 1588 in Ägypten, Syrien und Istanbul auf.

9 Hans Ulrich Krafft, Reisen und Gefangenschaft Hans Ulrich Kraffts. Hrsg. von K[onrad] D. Haßler, Stuttgart 1861. Hans Ulrich Krafft verbrachte in den siebziger Jahren des 16. Jahrhunderts einige Jahre in osmanischer Gefangenschaft.

10 Johann Wild, Neue Reysbeschreibung eines Gefangenen Christen ..., Nürnberg 1613, auch Nürnberg 1623.

11 Vgl. Michael Heberer, Aegyptiaca Servitus. Mit einer Einl. von Karl Teply, Graz 1967 (= Frühe Reisen und Seefahrten in Originalberichten, 6). Über Heberers Reisen und ihren politischen Hintergrund vgl. Thomas Freller, The life and adventures of Michael Heberer von Bretten, Malta 1997; Thomas Freller, Spies and Renegades, Malta 2004, S. $97 \mathrm{f}$.

12 Vgl. Beschreibung der Reisen des Reinhold Lubenau. Hrsg. von Wilhelm Sahm, 2 Bde, Königsberg 1914-1915.

$13 \mathrm{Zu}$ Lubenaus Geburt und Lebensweg vgl. die Einführung von Wilhelm Sahm in: Beschreibung (wie Anm. 12), Bd 1, S. IV-XIII. 
Schulzeit absolvierte Reinhold Lubenau beim Hofapotheker und Leibarzt des brandenburg-preußischen Herzogs Albert Friedrich, Jakob Montanus, eine Lehre. Im Haus des in Bologna promovierten und vielgereisten Montanus geriet der junge Reinhold Lubenau erstmals mit der inspirierenden Welt des mitteleuropäischen Humanismus in Berührung - eine Erfahrung die seinen zukünftigen Lebensweg entscheidend beeinflussen sollte. In den späten 1570er- und frühen 1580er Jahren führten ihn Verwandtenbesuche, Studien- und Ausbildungsreisen in verschiedene baltische Länder und deutsche Regionen. Familienkonflikte und eine bessere berufliche Perspektive als Apotheker und Leibarzt führten ihn nach Riga, Wilna und schließlich nach Wien. Dort schloss er sich - vorerst für ein Jahr - als Arzt und Apotheker der im Februar 1587 unter der Führung des neu ernannten kaiserlichen Orators (Botschafters) an der Hohen Pforte, Bartolomeus Betz, nach Konstantinopel abreisenden Gesandtschaft $\mathrm{an}^{14}$.

Die eineinhalb Jahre an der Metropole am Bosporus waren von verschiedensten Aktivitäten und einigen Reisen an die Schwarzmeerküste, in das Innere des kleinasiatischen Teils der Türkei und auf verschiedene griechische Inseln geprägt. Als nicht zuletzt aus konfessionellen Gründen die Spannungen mit dem katholischen, von Zeitgenossen als schwierig beschriebenen ${ }^{15}$, kaiserlichen Botschafter Bartolomeus Betz zunahmen, entschied sich der Protestant Lubenau im September 1588, den kaiserlichen Dienst zu quittieren. Entgegen den Anordnungen von Betz und mithilfe des englischen Gesandten an der Hohen Pforte, Eduard Burton, begab sich Lubenau heimlich an Bord einer Galeere einer vom neu ernannten Kapudan Pascha (»Kapudan Paşa «), Hassan Pascha geführten Inspektions- und Kaperfahrt. Sie soll im Zentrum dieses Beitrags stehen. Nach dem Ende dieser Reise schiffte sich Lubenau nach Venedig ein, besuchte einige weitere damals populäre Ziele der sich allmählich etablierenden Kavalierstour, bevor er im Oktober 1589 wieder im heimatlichen Königsberg eintraf und sich dort als Apotheker niederließ.

Versuchen wir im Folgenden zunächst Lubenaus Aufzeichnungen in einen Gesamtkontext des damaligen Geschehens zu stellen und seine Einblicke in die Situation der osmanischen Marine zu diskutieren, bevor wir uns im Detail mit seiner Fahrt beschäftigen. Globaler Hintergrund von Lubenaus Abenteuern im Mittelmeer ist ein von der Forschung mit "peripheralization $\ll^{16}$ oder Marginalisie-

$14 \mathrm{Zu}$ dieser Gesandtschaft vgl. Kaiserliche Gesandtschaften ans Goldene Horn. Hrsg. von Karl Teply, Stuttgart 1968, S. 304 f.

15 Vgl. Heberer, Aegyptiaca Servitus (wie Anm. 8), S. 301f.; Freller, The life (wie Anm. 11), S. 93f. Betz bat im Sommer 1588 selbst - unter Vorgabe gesundheitlicher Gründe - um seine Abberufung aus Konstantinopel. Vgl. Beschreibung (wie Anm. 12), Bd 2, S. 31 f.

16 Vgl. Michel Fontenay, The Mediterranean, 1500-1800. Social and Economic Perspectives. In: Hospitaller Malta (wie Anm. 3), S. 43-110. 
rung des Raums umschriebener Prozess, ohne dass sich dieses noch unmittelbar und dramatisch bemerkbar machte ${ }^{17}$. Im Gesamtkontext europäischer Politik sank jedoch die Bedeutung der nordafrikanischen und mediterranen Regionen zu Sekundärschauplätzen. Die Historiker Fernand Braudel, Kenneth M. Setton, David Abulafia, Maurice Aymard, Alexander H. de Groot oder Michel Fontenay haben ausführlichere Analysen eines ökonomischen und politischen Prozesses der »Entwertung « des Mittelmeerraums im 16. und 17. Jahrhundert zugunsten des Atlantiks durchgeführt ${ }^{18}$. Mit dem direkten Zugriff auf Indien und den asiatischen Raum durch den Handelsweg um das Kap der Guten Hoffnung und der Erschließung der Neuen Welt verlagerte sich im Verlauf des 16. Jahrhunderts das Zentrum des merkantilen Europa zunehmend von Italien und dem Mittelmeerraum nach Portugal, Spanien, England, Frankreich und den Niederlanden. Gleichzeitig wurde der zentrale und östliche Mittelmeerraum für diese Staaten zu einem politischen und ökonomischen Schauplatz zweiter Ordnung ${ }^{19}$. Neben den Staaten auf dem italienischen Stiefel war es vor allem die ehemalige Weltmacht Spanien, deren technologisches, ökonomisches und politisches Absinken - wenngleich es in einem eher schleichenden Prozess verlief - gerade im Mittelmeer spürbar wurde. Der Handel, die politischen Einflusssphären und das Netz der internationalen Kontakte und Austauschprozesse im Mittelmeer während des 17. und 18. Jahrhunderts wurden von England, den Niederlanden und Frankreich bestimmt ${ }^{20}$.

17 Für eine Untersuchung des türkischen Blickwinkels vgl. Stanford J. Shaw and Ezel Kural Shaw, History of the Ottoman Empire and Modern Turkey, vol. 1, Darmstadt 1985.

18 Vgl. im größeren Zusammenhang Immanuel Wallerstein, The Modern World-System, vol. 1: Capitalist Agriculture and the Origins of the European World-Economy in the Sixteenth Century, vol. 2: Mercantilism and the Consolidation of the European World-Economy, 1600-1750, New York, London 1974-1980. An technologischen und logistischen Beispielen wird diese Entwicklung aufgezeigt von John H. Pryor, Geography, Technology and War: Studies in the Maritime History of the Mediterranean 649-1571, Cambridge 1988; wegweisende, klassische Studie zur sozio-kulturellen, politischen und historischen Verortung der hier geschilderten Ereignisse im Mittelmeerraum bleibt Fernand Braudel, La Méditerranée et le monde méditerranéen à l'épogue de Philippe II., 2 t., hier zitiert ist die 2. Aufl., Paris 1966. Für die Veränderungen des 17. Jahrhunderts vgl. stellvertretend Kenneth M. Setton, Venice, Austria, and the Turks in the Seventeenth Century, Philadelphia, PA 1991. Für eine zusammenfassende Perspektive der Frühen Neuzeit vgl. Fontenay, The Mediterranean (wie Anm. 16), S. 43-110.

19 Vielleicht signifikantestes Ereignis und Fanal einer gefährdeten Zukunft war die Venedig im Oktober 1503 erreichende Nachricht, drei portugiesische Schiffe hätten über den Seeweg um das Kap der Guten Hoffnung 3000 quintali Pfeffer aus Indien nach Portugal transportiert. Diese Menge entsprach etwa der von den Venezianern in einem Jahr aus der Levante eingehandelten Menge an Pfeffer. Hier zitiert nach Fontenay, The Mediterranean (wie Anm. 16), S. 56.

20 Vgl. auch Setton, Venice, Austria (wie Anm. 18). 
$\mathrm{Zu}$ Lubenaus Zeiten waren die im zweiten Drittel des Jahrhunderts so engen Beziehungen zwischen dem Osmanischen Reich und Frankreich wieder etwas gelockert und primär auf Teile des französischen Hofes bzw. der Hugenotten beschränkt ${ }^{21}$. England sollte bald als strategischer osmanischer Geschäftspartner das von Bürgerkriegen geschüttelte Frankreich ersetzen ${ }^{22}$. 1580 erhielten die Engländer ähnliche Handelsprivilegien wie sie den Franzosen schon 1569 gewährt wurden.

$\mathrm{Zu}$ einem britisch-osmanischen Militärbündnis kam es indes nicht. 1585 sollten die von den Engländern mit der Hohen Pforte ausgehandelten Privilegien auch für die nordafrikanischen Fürstentümer gelten. Mehr noch als zuvor verlor mit dem massiven Eintreffen der Engländer und Niederländer im Mittelmeer die Vision einer »Heiligen Liga« gegen die Osmanen an Bedeutung ${ }^{23}$. Bezeichnenderweise war es der englische Vertreter an der Hohen Pforte, Eduard Barton, der Lubenau eine Passage in die Heimat verschaffte. Es waren ebenfalls Barton und französische Diplomaten, die einige Monate zuvor die Freilassung des oben erwähnten Galeerensklaven aus Heidelberg, Michael Heberer, erreichten ${ }^{24}$.

Trotz des ökonomischen Ausgreifens Europas nach Übersee und des damit einhergehenden strategischen und politischen Bedeutungsverlusts, wies das Wachstum des Mittelmeerhandels im 16. und 17. Jahrhundert noch dermaßen beträchtliche Steigerungsraten auf, dass zum Transport englische, flämische, französische und niederländische Schiffe unverzichtbar wurden. Bis zur Wende des 16. zum 17. Jahrhundert blieb der Mittelmeerraum für Europa der größte Umschlagplatz und Ex- und Importmarkt. Noch 1660 absorbierten etwa Ost- und Westindien nur etwa 10 Prozent der englischen Ausfuhren, während die Hälfte des Exports in den Mittelmeerraum floss ${ }^{25}$. Wirklich drastische Veränderungen

21 Michel Lesure, Les relations franco-ottomanes à l'epreuve des guerres de religion. In: L'Empire Ottoman, la Republique de Turquie et la France. Ed. par H. Batu et J.-L. Bacqué-Grammont, Istanbul 1986. $\mathrm{Zu}$ den geplanten gemeinsamen osmanisch-französischen maritimen Unternehmungen vgl. Dorothy M. Vaughan, Europe and the Turk, Liverpool 1954, S. 170-182.

22 Über die aggressive Ausdehnung des englischen und niederländischen Handelsimperiums in das Mittelmeer nach 1570 vgl. Richard T. Rapp, The Unmaking of the Mediterranean Trade Hegemony: International Trade Rivalry and the Commercial Revolution. In: The Journal of Economic History, 35 (1975), S. 494-525; Robert Davis, The Rise of the English Shipbuilding Industry in the Seventeenth and Eighteenth Centuries, London 1962; Holden Furber, Rival Empires of Trade in the Orient 1600-1800, Minneapolis, MN 1976.

23 Vgl. auch die diesbezüglichen Analysen von Paul Coles, The Ottoman Impact on Europe, London 1968, S. 98-120.

24 Heberer, Aegyptiaca Servitus (wie Anm. 8), S. 310f.; Freller, The life (wie Anm. 11), S. $93 \mathrm{f}$.

25 Vgl. Ralph Davis, England's Foreign Trade, 1660-1700. In: Economic History Review, $2^{\text {nd }}$ series, 7 (1954), S. 150-166. 
stellten sich erst ab der zweiten Hälfte des 17. Jahrhundert ein. Ähnliche Beobachtungen gelten für den zeitgenössischen französischen Handel.

Der Levantehandel war besonders hart von der Etablierung der neuen Atlantik-Routen betroffen. Unser Chronist Reinhold Lubenau deutet bei der Schilderung seines Besuchs von Alexandria im Oktober 1588 einige Effekte dieser Entwicklung an:

»Die Stadt [Alexandria] ist aber in ihrer Wurde nicht mehr so hoch als wol der Beruf, und der Nahme ist; den die Handlung itzo fast gahr abgenohmen, weil nunmehr die Spetzereien, Perlen, Edelgestein, Seiden und allerlei chinesische und indische Wahren aus India uber Mehr auf Lisbohn und Affrica herumb gehen, die vordem uber das Rote Mehr nachmahl auf dem Nilo gehen Alexandria und vollendt gehen Venedigk und also ferner seindt gefuhret worden. Und wan sich nach diese Zeit der venedische und frantzösische Coonsul sampt ihren Dienern, sowol derselben Nationen, Factoren und Liegern alhie nicht aufhilten, die ihre Fondigo und Kaufheuser haben und sonsten noch mit etlichen Freiheiten vom turckischen Keiser begabet seindt, so wahr es gahr ein elendes Wesen in dieser Stadt und verdreust dem Turcken nicht wenigk vielmehr aber den Venedigern, die durch diese Stadt ist aufkomen und reich worden, das nunmehr die Fahrt aus Hispanien umb Capo de Bone Sperantza erfunden, dadurch inen ein groser Abbruch geschicht, und nunmehr vollendt, weil die Hollender, Engelender, Frantzosen und andere Nationen sich derselben gebrauchen $^{26}$.«

Schiffsaufkommen und vernachlässigte Infrastruktur der Hafenanlagen spiegelten diese Situation wider ${ }^{27}$.

\section{Die osmanische Marine: Möglichkeiten und Grenzen}

Lubenaus Beschreibungen des baufälligen Zustandes vieler osmanischer Festungen und seine Hinweise auf das Alter verschiedener dort befindlicher Ausrüstungsgegenstände - etwa der Geschütze - dokumentieren die Fragilität des osmanischen Verteidigungssystems, besonders seine technische Schwerfälligkeit und zunehmende Rückschrittlichkeit. In Negroponte (Évvioia/Euböa) hatte der Königsberger im September 1588 die Gelegenheit, Hassan Pascha auf seinen Inspektionstouren zu den Festungen zu begleiten; gemäß seinen Beobachtungen »wahren in beiden Festungen etlich Hundert groser, gegossener Stuck Geschutz,

26 Beschreibung (wie Anm. 12), Bd 2, S. 216f.

27 Ebd., S. 213. 
die zum Theil gahr alt« (»Veneziano«) $)^{28}$. Viele von ihnen waren im Abendland hergestellt worden: »Habe auf einem gefunden die Jahrzahl 1423 und einen Nahmen darauf Jacobus Ferrarius me fecit, [...] Ich halte es davor, das es der Maltheser gewesen und ihnen zu Rhodis genohmen sei. Auf einem stundt Johannes Malvesius Author, dasselbe wahr gahr kunstlich mit Laubwergk umb un umb, und die Jahrzahl 1530. Auf einem eine Schlange und ein Kindt im Munde; darauf stundt Philippus Rex « ${ }^{29}$.

Konzentrieren wir uns im Folgenden auf die Einblicke, welche Lubenau in den Zustand und die "Betriebsabläufe« der osmanischen Marine des späten 16. Jahrhunderts liefert ${ }^{30}$. Die osmanische Marine im Mittelmeer hatte sich in den Jahrzehnten zuvor grundlegend verändert ${ }^{31}$. War sie im 15. und frühen 16. Jahrhundert strategisch mit den Landtruppen untrennbar verknüpft, entwickelte sie sich - teilweise angelehnt an christliche Vorbilder - im Verlauf des 16. Jahrhunderts mehr und mehr zu einer eigenständigen Einheit. Nach wie vor blieb allerdings die kombinierte Land- und Seeoperation eine Spezialität der Osmanen $^{32}$. Mit der Schaffung der nordafrikanischen Satellitenfürstentümer Tri-

28 Ebd., S. 175. Zu Hassan Pascha »Veneziano« vgl. u.a. Noel Malcolm, Agents of Empire: Knights, Corsairs, Jesuits and Spies in the Sixteenth-Century Mediterranean World, London 2015, S. 370 f. und S. 392.

29 Beschreibung (wie Anm. 12), Bd 2, S. 175.

30 Eine detaillierte Geschichte der osmanischen Marine des 16. Jahrhunderts ist bisher ein Desiderat der Forschung. Wertvolle Einblicke geben die Monografien von Carlo M. Cipolla, Guns, Sails and Empire, New York 1965; Colin J. Imber, The Navy of Süleyman the Magnificent. In: Archivum Ottomanicum, 6 (1980), S. 210-282; Palmyra Brummet, Ottoman Seapower and Levantine Diplomacy in the Age of Discovery, New York 1994; Andreas Rieger, Die Seeaktivitäten der muslimischen Beutefahrer als Bestandteil der staatlichen Flotte während der osmanischen Expansion im Mittelmeer im 15. und 16. Jahrhundert, Berlin 1994; John F. Guilmartin, Gunpowder and Galleys. Changing technology and Mediterranean warfare at sea in the sixteenth century, Cambridge [u.a.] 1980; Groot, The Ottoman Threat (wie Anm. 3), S. 199-254; Salvatore Bono, I corsari barbareschi, Torino 1964; Salvatore Bono, Naval Exploits and Privateering. In: Hospitaller Malta (wie Anm. 3), S. 351-398; und kürzlich Adrian Tinniswood, Pirates of Barbary. Corsairs, Conquests and Captivity in the Seventeenth-Century Mediterranean, London 2010.

31 Die entsprechenden Kapitel über die osmanische Expansion und Militärmaschinerie des 16. Jahrhunderts im Bd 1 von Stanford J. Shaw und Ezel Kural Shaws History of the Ottoman Empire (wie Anm. 17) sind in ihren Details zur Marinegeschichte der Osmanen nicht immer präzise. Einen genaueren Einblick in die hier beschriebene Epoche liefern Halil Inalcik, The Ottoman Empire. The Classical Age 1300-1600, London 1973, und die relevanten Passagen in Halil Inalcik, The Social and Economic History of the Ottoman Empire, Cambridge 1994.

32 Wertvolle Einblicke in die unmittelbar zeitgenössischen Strukturen gibt Caroline Finkel, The Administration of Warfare: The Ottoman Military Campaigns in Hungary 1583-1606, Wien 1988. 
polis, Tunis und Algier entstand eine neue Lage ${ }^{33}$. Die osmanischen Flotten und Verbände hatten nun auch im Zentrum des Mittelmeers die Möglichkeit, auf relativ sichere Häfen und Versorgungsstationen zurückzugreifen. Damit war die Gelegenheit $\mathrm{zu}$ weit in den westlichen Mittelmeerraum greifenden Operationen gegeben; nicht selten handelte es sich dabei um kombinierte nordafrikanischosmanische Unternehmungen ${ }^{34}$.

Der Königsberger Chronist ist Zeuge der flexiblen Integration der Flotten der nordafrikanischen Fürstentümer in das osmanische Gesamtkonzept der Expansion der See-Kontrolle vom östlichen in das westliche Mittelmeer. Die Grenzen zwischen Korsarenexkursionen und »offiziellen« militärischen Operationen waren dabei häufig fließend. Große personal- und materialvernichtende Seeschlachten sollten möglichst vermieden werden; Ziele waren vor allem die Kaperung wertvoller Fracht und Landrazzien zur Erbeutung von Sklaven und Gütern. Die Forschung hat in diesem Zusammenhang auf einen wesentlichen Unterschied zwischen der Effizienz, dem technischen Niveau und der Stärke der osmanischen Landtruppen und der Marine hingewiesen ${ }^{35}$. Die Schiffstypen der osmanischen Kriegsmarine und ihre Mannschaften besaßen niemals die Schlagkraft ihrer venezianischen, genuesischen oder maltesischen Widersacher ${ }^{36}$. Im Allgemeinen wurde eine vier- oder fünffache Überlegenheit als nötig erachtet, um Seeschlachten erfolgreich führen zu können. Nur sehr wenige größere Seeschlachten zwischen christlichen und islamischen Kräften endeten im 16. Jahr-

33 Ciro Manca, Il modello di sviluppo economico delle città marittime barbaresche dopo Lepanto, Napoli 1982. Speziell zur Bedeutung Algiers vgl. John B. Woolf, The Barbary Coast. Algeria under the Turks, New York 1979; zu Tunis vgl. Taoufik Bachrouch, Formation sociale barbaresque et pouvoir politique à Tunis, Tunis 1977.

34 Zur Effizienz und militärischen Rolle der Streitkräfte der nordafrikanischen Satelliten und ihrer Korsaren vgl. Godfrey Fisher, Barbary Legend, Oxford 1957; Peter Earle, Corsairs of Malta and Barbary, London 1970; Jacques Heers, The Barbary Corsairs - Warfare in the Mediterranean 1480-1580, London 2003; Michel Fontenay et Alberto Tenenti, Course et piraterie méditerranéennes de la fin du Moyen Age au début du XIXe siècle. In: Course et Piraterie, 18e Colloque International d'Histoire maritime, San Francisco 1975, Paris 1975, S. 78-131; Michel Fontenay, La place de la course dans l'économie portuaire: l'exemple de Malte et des ports barbaresques. In: Annales E.S.C., 43 (1988), S. 1321-1347.

35 Für eine allgemeine Perspektive vgl. Groot, The Ottoman Threat (wie Anm. 3), S. 199-254. Detailliertere Untersuchungen zu den Land- und Seestreitkräften in der hier behandelten Epoche sind zu finden in Thomas Scheben, Schwendi, Montecuccoli, Kinsky: Analysen der Osmanischen Kriegsmacht vom 16. bis zum 18. Jahrhundert. In: CIEPO - VII. Symposium Actes (Istanbul 1994), und Thomas Scheben, A State with an Army - An Army with a State? - The Ottoman Power Machine. In: The 1565 Ottoman Malta Campaign Register (wie Anm. 1), S. 13-81.

36 Vgl. Scheben, A State (wie Anm. 35), S. 62. 
hundert mit einem Erfolg der Letzteren; dazu zählten etwa Preveso 1538 und Djerba 1560.

Mehr noch als der vom kalabresischen Konvertiten und Galeerensklaven zum osmanischen Großadmiral (»Kapudan-i derya«) aufgestiegene Uluç Alī (»Occhiali«, 1519-1587) war Sokollu Mehmet Pascha (ca. 1506-1579) treibende Kraft beim Versuch, die osmanische Flotte nach dem Desaster von Lepanto wiederherzustellen. Wie Uluç Alī entstammte der in Serbien geborene Sokollu Mehmet bescheidenen Verhältnissen. Seine Karriere im osmanischen Militär und der Verwaltung erreichte 1565 mit seiner Ernennung zum Großwesir ihren Höhepunkt. Vor allem unter Sultan Selim II. (1566-1574) besaß er die Freiräume, eine energische Reformpolitik im Heer anzustoßen und die osmanische Politik zu lenken. Zusätzlich zu den alten Arsenalen und Werften am Goldenen Horn entstanden neue Anlagen in der Nähe der Gärten des Sultans und des Serails. Weitere Werften, auf denen Galeeren, Fusten und Galeoten gebaut wurden, befanden sich in Varna, Burgas, Igneada Burnu, Midie, Kefken, Kerpe, Samsun, Semendria, Izmit, Gemlik, Yalova, Rhodos und Antalya.

Bekannter Vorteil der Osmanen blieb ihre große Flexibilität, ihre erstaunlich durchlässige und damit für den Einzelnen, besonders für Konvertiten, motivierende militärische Hierarchie und eine geschickte Ausnutzung von Ressourcen. Die Stärke der osmanischen Militärmaschinerie und der Flotte waren die durch eine durchlässige Hierarchie hervorgerufene hohe Motivation und durch persönliches Vorbild und Führungsstärke angeregte Disziplin unter den Soldaten $^{37}$.

Lubenaus Notizen eröffnen uns in diesem Zusammenhang interessante Einblicke in das erst in den letzten Jahrzehnten vermehrt in das Blickfeld der Wissenschaft geratene Phänomen des Konvertitenwesens ${ }^{38}$. Nicht selten trafen Lubenau und die anderen oben genannten Tagebuch- und Reiseberichtsschreiber im Osmanischen Reich oder in den nordafrikanischen Fürstentümern auf zum islamischen Glauben konvertierte Deutsche, Franzosen, Engländer oder Polen, etwa jener von Lubenau im August 1587 in Konstantinopel getroffene »Michel von Strasburgk, der zum Turcken worden wahr « ${ }^{39}$, oder den ebenfalls zum »Turcken« gewordenen

37 Vgl. die zeitgenössischen Kommentare des Malteserritters Georg Schilling von Canstatt an den deutschen Großprior vom 25.1.1539. Abgedr. in: Deutsche Johanniterbriefe aus dem sechszehnten Jahrhundert. Hrsg. von Heinrich Meisner. In: Zeitschrift für die Geschichte des Oberrheins, N.F., 10 (1895), S. 565-631, hier S. 630. Vgl. auch Uberto Foglieta, De causis magnitudinis Turcarum Imperii, Rostock 1594, S. 3 f.

38 Vgl. die wegweisenden Studien von Bartolomé Bennassar et Lucile Bennassar, Les chrétiens d'Allah. L'histoire extraordinaire des renégats. XVIe-XVIIe siècles, Paris 1989.

39 Beschreibung (wie Anm. 12), Bd 2, S. 20. 
»Goldschmidt, Hans von Nurenbergk genannt « $^{40}$. Auch in Alexandria stand Lubenau im Kontakt mit »einem deutschen Renegaten [Konvertiten], so sich sonsten fur einen Dolmetschen ausgibt Hans aus Beuren der Geburt « ${ }^{41}$. Nach ihrer Konvertierung verfügten die ehemaligen Christen hinsichtlich Status und Aufstieg über die gleichen Chancen wie ihre »altgläubigen« islamischen Glaubensbrüder. Angesichts eines steten Mangels an militärischen und technischen Spezialisten musste der osmanischen Führung stets daran gelegen sein, für einen Nachschub von Konvertiten und ausländischen Fachkräften offen zu sein.

Die Notizen Lubenaus geben darüber hinaus wertvolle Hinweise, inwiefern es sich bei der landläufigen Annahme, die Ruderer der Galeeren hätten durchweg den Status von Sklaven gehabt, um einen Irrtum handelt. Selbst bei den zeitgenössischen christlichen Flotten der Florentiner, Malteser, Genuesen oder Venezianer gab es die »buonavoglie«; Männer, die ihre Schulden durch Galeerenarbeit abarbeiten wollten oder mussten ${ }^{42}$. Der Anteil der Sklaven auf den Galeeren orientierte sich am Personalreservoir der jeweiligen Staaten. In den kleineren maritimen Seemächten, etwa dem Staat der Johanniter auf Malta oder des Großherzogtums Florenz, war der Anteil an Sklaven auf den Schiffen höher; das an Arbeitskräften reiche Osmanische Reich dagegen konnte seine Galeeren mit einem hohen Anteil von Freiwilligen bemannen. Die osmanische Führung bevorzugte aus verschiedenen Gründen den Einsatz von im Allgemeinen gut bezahlten und mit einem Anteil an der Beute belohnten Freiwilligen. Sie mussten zum einen nicht permanent bewacht werden und konnten bei Landoperationen gleichzeitig als Kriegsknechte herangezogen werden ${ }^{43}$.

Wie im Fall der von Lubenau beschriebenen Fahrt des Hassan Pascha »Veneziano« im Spätsommer 1588 fuhren auf den osmanischen Schiffen Kontingente von gut ausgebildeten Janitscharen und »Sipahi «44. Die »Sipahi« waren ursprünglich mit schweren Bögen ausgestattete Kavalleristen, konnten aber auch als Infanteristen eingesetzt werden. Bezüglich Reichweite, Genauigkeit und Schussfrequenz übertrafen ihre schweren Bögen jede Handfeuerwaffe bzw. Muskete des 16. Jahrhunderts. Wie wir anlässlich von Lubenaus Beschreibung der Razzia süd-

40 Ebd., S. $42 f$.

41 Ebd., S. 215.

42 Am Beispiel der Marine des Johanniterordens analysiert von Joseph Muscat, Galley Slaves at work, Malta 2007.

43 Zum Personal auf den osmanischen Galeeren des 17. Jahrhunderts vgl. Michel Fontenay, Chiourmes turques au XVIIe siècle. In: Le genti del mare Mediterraneo. A cura di Rosalba Ragosta, 2 P., Napoli 1981, hier P. 2, S. 877-903.

44 Zur Rolle der Janitscharen und »Sipahi« vgl. Nahoum Weissmann, Les Janissaires, Paris 1964; Godfrey Goodwin, The Janissaries, London 1994. 
lich von Barcelona Anfang Oktober 1588 sehen werden, waren es die Janitscharen und »Sipahi«, die mit Spezialgeräten und mitgeführten Eseln und Pferden die gut vorbereiteten Exkursionen in das Landesinnere unternahmen ${ }^{45}$.

Augenzeugen wie André Thevet ${ }^{46}$, Ogier Giselin de Busbeq ${ }^{47}$, Antoine Geuffroy $^{48}$, Pierre Belon ${ }^{49}$ oder Nicolas de Nicolay ${ }^{50}$ berichten in der Mitte des 16. Jahrhunderts, dass nicht nur in den Geschützgießereien in Konstantinopel zahlreiche ausländische Spezialisten beschäftigt waren; auch die Werften und die allein über 50 Galeeren Platz und Schutz bietenden Arsenale und Docks von Galata beschäftigten griechische, italienische und französische Techniker, Ingenieure und Zimmerleute. Ihr technisches Wissen trug wesentlich zum Aufbau und zur stetigen Verbesserung der Schiffe einer Macht bei, die ursprünglich aus dem Nukleus eines Steppenvolkes erwuchs. Wir lesen mehrmals, wie der ausgebildete Apotheker Lubenau während seiner Mittelmeerreise von Offizieren mit großzügigen Versprechungen zur Aufnahme von osmanischen Diensten angeregt wurde ${ }^{51}$. In der Tat war die Gesundheitsvorsorge ein wesentlicher Faktor auf Seereisen. Nicht behandelte Infektionen konnten sich angesichts der beengten Verhältnisse besonders rasch ausbreiten, krankes Personal konnte gesamte Feldzüge zum Scheitern bringen ${ }^{52}$.

Im Allgemeinen taten in der Marine der Osmanen und nordafrikanischen Fürstentümer die ähnlichen Schiffstypen Dienst wie bei den christlichen Mächten. Im 16. Jahrhundert waren dies Galeeren, Karacken, Galeonen, Fusten und frühe Formen von Schebecken und Brigantinen ${ }^{53}$. Während in der osmanischen Marine kaum die vor allem von den Venezianern verwendeten, bis zu 70 Meter langen Galeassen - eine Mischung zwischen Segel- und Ruderschiffen - Dienst taten, hören wir im Fall der nordafrikanischen Satelliten häufig von Fusten mit ihren nur 12 bis 18 Riemenpaaren, einem Lateinsegel und zwei oder drei Kanonen und

45 Beschreibung (wie Anm. 12), Bd 2, S. 198.

46 André Thevet, Cosmographie de Levant, Paris 1554, S. 50 f. Vgl. auch André Thevet, Cosmographie Universelle, Paris 1575.

47 Vgl. Ogier Giselin de Busbeq, Vier Briefe aus der Türkei, Erlangen 1926.

48 Antoine Geuffroi, Estat de la court du Grand Turc, Paris, Antwerpen 1542.

$49 \mathrm{Vgl}$. Pierre Belon, Les observations de plusieurs singularitez et choses memorables trouvées en Grèce, Asie, Judée, Egypte, Arabie et autres pays estranges, Paris 1553.

50 Nicolas de Nicolay, Le Navigationi et Viaggi nella Turchia, Antwerpen 1576.

51 Beschreibung (wie Anm. 12), Bd 2, S. 137.

52 Vgl. am Beispiel der maltesischen Galeeren Joseph Muscat, Medical and Sanitary Aspects on Maltese Galleys. In: Sacra Militia, 2 (2003), S. 49-60.

$53 \mathrm{Zu}$ den damals in Gebrauch befindlichen Schiffstypen vgl. Michele Calegari, Legname e costruzioni navali nel Cinquecento. In: Guerra e commercio nell'evoluzione della marina genovese tra XV e XVII secolo, 2 P., Genua 1970-1973, hier P. 2, S. 79-148. 
von Galeoten, einer verkleinerten Form der Galeere, mit ihren zwischen 18 und 22 Riemenpaaren. Letztere Schiffstypen wurden damals auch häufig von Korsaren und Piraten benutzt, sie waren leicht und schnell, boten die Möglichkeit, sich in seichten Buchten zu verstecken und auch Flüsse $\mathrm{zu}$ befahren. Auf christlicher und islamischer Seite war in Seeschlachten die Hauptfeuerkraft auf den Karacken konzentriert, hochbordige Dreimaster, deren Rumpf nicht in Klinker-, sondern Kraweeltechnik gebaut war. Sie waren damit schneller und besser mit Geschützluken auszustatten.

Anhand der Beschreibungen der Augenzeugen und wenigen aus der Epoche existierenden Schiffsresten tendieren Marinehistoriker zu der Annahme, dass die islamischen und nordafrikanischen Schiffe insgesamt eine leichtere Bauweise als ihre christlichen Gegenstücke aufwiesen. Weiteres besonderes Merkmal der Zusammensetzung osmanischer Flotten war ihr höherer Anteil von leichten Schiffstypen im Vergleich zu den christlichen Flotten. Die von Lubenau beschriebene Expedition, mit ihrem hohen Anteil von Galeeren, machte hier eine Ausnahme. Diese Zusammensetzung der Flotte stand im Kontext des Auftrags von Hassan Pascha, in relativ kurzer Zeit große Distanzen - auch in feindlichen Gewässern - zurückzulegen. Im Fall von Gefahr oder anderweitigen Befehlen sollte umgehend eine Rückkehr zu den Heimathäfen möglich sein. Eine andere Stärke der osmanischen Marine - und eng verknüpft mit ihrer strategischen Ausrichtung zur Unterstützung von Landoperation - war ihre Effizienz beim Transport von großen Truppenkontingenten. Dies stellten gerade im 16. Jahrhundert die Eroberung des Mameluckenreichs von Ägypten (1517), der Siegeszug entlang der östlichen Adriaküste, die Eroberungen bzw. Rückeroberungen von Tripolis (1551) und Tunis (1574) oder die erfolgreiche Anlandung von über 30000 Soldaten anlässlich der Belagerung der Festungen Maltas (1565) unter Beweis.

Für die gesamte Zeit der hier beschriebenen Inspektionsreise und Kaperfahrt fuhr unser Chronist auf einer osmanischen Galeere. Ähnlich wie bei den Galeeren der christlichen Mächte hatten auch auf den osmanischen Galeeren Mobilität und Geschwindigkeit oberste Priorität ${ }^{54}$. Das bedeutete, dass über 90 Prozent der Deckfläche von den Bänken der Rudersklaven besetzt war. Die venezianischen und osmanischen Galeeren galten als die schnellsten derartigen Schiffstypen, jenen der Spanier, Franzosen, Florentiner, Genuesen oder Malteser überlegen ${ }^{55}$.

$54 \mathrm{Zu}$ den Vor- und Nachteilen der Galeeren des 16 Jahrhunderts vgl. Frédéric Chapin Lane, Venetian Ships and Shipbuilders of the Renaissance, Baltimore, MD 1934; Jack Beeching, The Galleys at Lepanto, London 1982; Francisco-Felipe Olesa Munido, La Galera en la navegacíon y el combate, 2 P., Madrid 1971.

55 Scheben, A State (wie Anm. 35), S. 64; vgl. auch Chapin Lane, Venetian Ships (wie Anm. 54), S. $373 \mathrm{f}$. 
Der flache Kiel und das niedrige Deck begrenzten die Segelqualitäten und Wetterfestigkeit der Galeeren. Mit günstigen Windverhältnissen und unter Einsatz der Ruder ließ sich über eine kurze Zeit eine Geschwindigkeit von 12 Knoten erreichen. Unter normalen Wetterverhältnissen konnte eine Geschwindigkeit pro Stunde von etwa 3 Knoten über einen längeren Zeitraum aufrechterhalten werden. Um die Arbeitskraft und Energie der Galeerenruderer für Notfälle von Feindkontakt und schlechten Wetterverhältnissen zu sparen, versuchte man soweit es ging, auf die Windkraft, also Segel zurückzugreifen. Die primär auf den Segeln des Hauptmasts liegende Last der Fortbewegung bot wenig mehr als 1,5 oder zwei Knoten Geschwindigkeit. Die bei einer Mannschaftsstärke von etwa 150 Ruderern angenommene Spitzengeschwindigkeit betrug bei Windstille etwa 7 Knoten. Diese Geschwindigkeit konnte für maximal eine halbe Stunde aufrechterhalten werden $^{56}$. Lubenau gibt einen Einblick in den zur Aufrechterhaltung dieser Geschwindigkeit nötigen Kraftaufwand. Auf der Fahrt entlang der libyschen Küste notierte er im Oktober 1588:

\footnotetext{
»Kegen den Abendt begunt es gantz stille zu werden und musten die Sclaven rudern [...] Es wahr ein junger Italiener erst neulich auf die Galleen komen und hardt bei meiner Bancken angeschmidet. Denselben lis ich an die Seite sitzen, setzte mich zum Ruder und half zihen. Ich ruderte aber kaum eine Viertelstunde, da thatem mihr die Ackseln so wehe, das ich sie nicht wol ruren kuntte, und hatte grose Blasen in die Hende bekomen ${ }^{57}$."
}

Schiffsstruktur, Bemannung und Bewaffnung setzten die militärische Verwendbarkeit von Galeeren, Galeoten und Galeassen deutlich von anderen Schiffstypen ab. Die osmanischen Galeeren - wie ihre venezianischen, spanischen, genuesischen oder maltesischen Gegenstücke - waren nach der Abschaffung des Rammsporns nicht dazu ausgelegt, gegnerische Schiffe $\mathrm{zu}$ versenken, sondern wurden vor allem zum Kapern eingesetzt. Die vor allem an Bug und Heck konzentrierte Feuerkraft sollte sich auf das Ausschalten des feindlichen Widerstands und damit die Vorbereitung des Enterns konzentrieren. Kanonenfeuer hatte das Ziel, die Mobilität des Feindes einzuschränken und dessen Entkommen zu verhindern. Diese Feuerkraft darf jedoch auch noch für das Ende des 16. Jahrhunderts nicht überschätzt werden. Zwar hören wir von venezianischen Geschützmeistern, deren Kanonen den Gegner aus fünfhundert Metern trafen, dies waren jedoch Ausnahmen. Im Allgemeinen spielten sich diese Duelle der Kanoniere auf hundert oder weniger Metern Distanz $\mathrm{ab}^{58}$.

56 Scheben, A State (wie Anm. 35), S. 65-68.

57 Beschreibung (wie Anm. 12), Bd 2, S. 209.

$58 \mathrm{Zu}$ den Einsatzmöglichkeiten der Galeeren am Ende des 16. Jahrhunderts vgl. neben den oben genannten Monografien von Munido, Muscat und Fontenay die zeitgenössischen Beschreibungen 
$\mathrm{Zu}$ Zeiten Lubenaus waren die Galeeren meist mit einem am Bug montierten schweren Geschütz und zwei oder drei leichteren Modellen ausgestattet. Dieses Hauptgeschütz konnte über 2000 Kilogramm wiegen, fünf Meter lang sein und dreißig Kilo schwere Kugeln verschießen. Zwar zeigen verschiedene Gemälde und Zeichnungen des späten 17. und des 18. Jahrhunderts an den erhöhten Positionen am Heck und den Seitenwänden angebrachte, bis zu einem Dutzend drehbare, leichte, gehacktes Eisen und Blei verschießende Geschütze, diese waren jedoch im 16. Jahrhundert noch die Ausnahme.

Im Allgemeinen wurde im 16. Jahrhundert noch nicht zwischen Land- und Seekanonieren unterschieden. Dies leuchtet im Besonderen bei den kombinierten Land- und Seeoperationen der Osmanen ein. Hier mussten Kanoniere (»topçu«, »humbaraci«) sowohl bei den See- als auch bei den folgenden Landoperationen ihren Dienst tun. Genauere Untersuchungen zur Ausbildung der in den Trainingskasernen von Gallipoli (Gelibolu) stationierten Kräfte sind bisher ein Desiderat der Forschung; hier scheinen Kanoniere speziell auf den Dienst auf See unterwiesen worden zu sein.

Häufig berichtet Lubenau, inwiefern die von Hassan Pascha geführte Flotte, etwa beim Verlassen der Dardanellen oder des Hafens von Napoli de Romania (Návplion) die vollständige Schlachtordnung einnahm. Das Üben der fest eingespielten Positionierung der einzelnen Schiffe nach Geschwindigkeit, Feuerkraft und Taktik war wesentliche Voraussetzung für das Bestehen einer Seeschlacht in einem Zeitalter, in dem auch maritime Schlachtordnungen noch sehr schematisch und unflexibel waren. Der Mangel an Geschützen an den Flanken der Galeeren, die Verwundbarkeit durch feindliches Feuer, die Gestalt der Zielfläche sowie die Position der eigenen schweren Feuerwaffen führten zu einer Taktik, die zunächst einen Frontalangriff und dann ein auf kurzer Distanz schräges Anlaufen zum Entern favorisierten. Attacken auf einzelne große "Rundschiffe« erfolgten in konzentrischer Form. d.h. das feindliche Schiff wurde von den Galeeren eingekreist und dann von allen Seiten angegriffen. Im Fall einer Verteidigung zogen sich die Galeeren in eine runde bzw. sternförmige Formation zurück, mit dem Bug und der dort positionierten schweren Artillerie den Gegner anvisierend.

Auch wenn Galeeren durch die Ruderkapazitäten wetterunabhängiger als reine Segelschiffe waren, hatten sie den mediterranen Windverhältnissen Tribut zu zollen. Die häufigsten Winde des Mittelmeerraums blasen aus nordöstlicher und nordwestlicher Richtung; dementsprechend gestalten sich Passagen von Nor-

von Hieronymus Zöber, Warhafftige/vollkommene und grundeliche Beschreybung derer Geschichten [...] des Türkischen Kriegsvolcks ..., Dillingen 1567, ohne Seitenzählung; Joseph Furtenbach, Architectura Navalis, Ulm 1629. 
den nach Süden bzw. von Westen nach Osten einfacher, als in entgegengesetzter Richtung. Ost-West-Passagen konnten im Allgemeinen doppelt so schnell durchgeführt werden, wie umgekehrt. Bezeichnenderweise legte die Flotte Hassan Paschas Anfang Oktober 1588 die Passage von der valencianischen Küste zum Golf von Tunis - mit Unterstützung von Ruderarbeit - in kaum zwei Tagen zurück ${ }^{59}$. Die Fahrten von Sardinien nach Korsika bzw. nach Marseille gestalteten sich weitaus schwieriger ${ }^{60}$. Aufgrund ihrer Untiefen und schlechteren Anker- und Versorgungsmöglichkeiten wurde ein Inselspringen in der Ägäis den Fahrten entlang der ägyptischen und libyschen Küsten vorgezogen. Auch hierzu liefert das Tagebuch des Reinhold Lubenau wertvolle Einblicke, wenn er beispielsweise von den schlechten Versorgungsmöglichkeiten entlang der Kyrenaika und Syrte berichtet ${ }^{61}$.

Schwachpunkt der osmanischen Marine blieb der Mangel an ausgebildeten Seeleuten und Marinesoldaten. Das Osmanische Reich war zwar in der Lage, in relativ kurzer Zeit die durch die verheerende Niederlage von Lepanto verlorenen Schiffskapazitäten und auch die Artillerie zu ersetzen, nicht aber den Personalverlust $^{62}$. Als Resultat wurde für eine längere Zeit auf Operationen großer Flottenverbände verzichtet. Im Osmanischen Reich des 16. Jahrhunderts existierte keine Kadettenschule oder Marine-Akademie als Schmiede zukünftiger Generationen von Marinepersonal. Das Erlernen von Kampftechniken auf See und von nautischen Fähigkeiten war ein langjähriger Erfahrungsprozess. Kam es durch Seeschlachten oder anderweitigen Gründen zu großen Verlusten an erfahrenem Seepersonal war dies ein entscheidender Nachteil. Die Heranziehung unerfahrenen Personals machte die Flotte wesentlich anfälliger für Niederlagen und weniger manövrierfähig in schwierigen Wetterverhältnissen. Die von Lubenau als Augenzeuge beschriebene Unternehmung des Hassan Pascha zu einer Vielzahl von Mittelmeerhäfen und verschiedenen Gewässern mit unterschiedlichen Wind- und Wasserverhältnissen, scheint neben ihren Inspektions- und Kaperabsichten auch den Charakter einer Trainingsfahrt besessen zu haben.

59 Beschreibung (wie Anm. 12), Bd 2, S. $198 \mathrm{f}$.

60 Vgl. ebd., S. $190 f$.

61 Ebd., S. $210 \mathrm{f}$.

62 Zur überschätzten Bedeutung der osmanischen Verluste bei Lepanto vgl. Andrew C. Hess, The Battle of Lepanto and its Place in Mediterranean History. In: Past and Present, 57 (1972), S. 53-72; Roger Charles Anderson, Naval Wars in the Levant 1559-1853, Liverpool 1952; Groot, The Ottoman Threat (wie Anm. 3), S. 199-203. 


\section{Maritime Basen und Operationsfelder}

Werfen wir vor der detaillierten Beschäftigung mit Lubenaus Tagebuch einen abschließenden Blick auf die Rolle der osmanischen Flotte am Ende des 16. Jahrhunderts im Gesamtkontext des Mittelmeergeschehens. Angesichts der sozioökonomischen Struktur, historischen Tradition und geografischen Verortung des Osmanischen Reiches wurde die Marine der Osmanen häufig als verlängerter Arm des Landheeres bezeichnet. Obwohl die Sultane im 15. Jahrhundert - speziell Mehmet II. (1451-1481) - eine umfangreiche und relativ schlagkräftige Flotte bauen ließen, verrät auch noch in den folgenden Jahrhunderten die militärische Strategie eine Konzentration auf den Landkrieg. Auch die größeren maritimen Operationen zur Eliminierung der christlichen Korsarenstützpunkte und der von den feindlichen Mächten genutzten Häfen und Inseln Rhodos (1522), Zypern (1570/71), Malta (1565) oder Kreta (1656-1669) waren ohne den Einsatz von umfangreichen Kontingenten von Landtruppen nicht durchzuführen. Für die im Verlauf des Jahrhunderts von spanischen Kräften attackierten Städte Tunis, Tripolis, Goletta, Algier, Biserta oder Mahdia war diese Gewährleistung einer reibungslosen maritimen Versorgung daher von entscheidender Bedeutung. Tripolis war den Johannitern erst 1551 entrissen worden, Tunis den Spaniern erst wieder $1574^{63}$. Hassan Paschas Inspektionsreise besaß insofern auch für die Koordination der Logistik und strategischen Planungen der Osmanen eine wichtige Rolle ${ }^{64}$.

Wie bereits angedeutet, war die osmanische Flotte im Allgemeinen in ein strategisches Konzept eingebunden, das ihr die Aufgabe des Transports von Truppen, Waffen und Versorgungsmaterial für die Landoperationen zuordnete. Diese auffallende Vernachlässigung der Flotte als eigenständige operative Einheit reflektiert sich zum einen in der häufigen Unterlegenheit der großen osmanischen Kriegsschiffe gegenüber den Gegenstücken der Venezianer, Genuesen oder Spanier, zum anderen darin, dass zunächst keine eigenständige Admiralität für die Galeerenflotte geschaffen wurde. Vereinfacht formuliert bestand die unter dem Kommando des Sultans stehende Kriegsflotte aus zwei Teilen: die regulären Verbände und die irregulären oder semi-irregulären Verbände der Korsaren. Dies

$63 \mathrm{Zu}$ den militärischen Operationen und zur osmanischen Expansion in Nordafrika vgl. André Clot, Suleiman the Magnificent, London 1992; Soliman le Magnifique. Actes du Colloque de Paris, Paris 1992.

64 Einzelaspekte und Strategie der militärischen Kriegführung der Osmanen in der frühen Neuzeit werden untersucht von Vernon J. Parry and Malcolm E. Yapp, War, Technology and Society in the Middle East, London [u.a.] 1975; vgl. auch Thomas M. Barker, Double Eagle and Crescent, New York 1967. 
entsprach der Situation der Landstreitkräfte. Die irregulären Verbände und deren Operationen dienten häufig als Trainingsfelder und Personalpool für die Mannschaften der regulären Flotten. Betrachtet man in der Gesamtschau die maritimen Unternehmungen des Osmanischen Reichs im Mittelmeer des 16. Jahrhunderts, operierten reguläre und irreguläre Verbände häufig zusammen. Die Flotten der Korsaren spielten dabei häufig die Rolle der leichten Kavallerie der Landheere; sie attackierten die Versorgungslinien des Feindes, zerstörten dessen Logistik und blockierten Nachschubwege.

Die von Lubenau beschriebene Fahrt Hassan Paschas mit seinen über $20 \mathrm{Ga-}$ leeren in das nordwestliche Mittelmeer stellte einen Sonderfall dar. Hintergrund war zum einen der von dem deutschen Chronisten erwähnte Inspektionscharakter, zum anderen die aufgrund der damaligen Konzentration der spanischen Kräfte im Atlantik gebotene Möglichkeit einer Erkundungs- und Kapertour in den iberisch beherrschten Teil des Mittelmeers. Sie war in diesem Kontext ein wertvoller »Testlauf« osmanischer Möglichkeiten und zur Feststellung eventueller gegnerischer Schwachstellen und Defizite. Dass der Großteil der spanischen Flotte damals im Atlantik beschäftigt war, machte den Zeitpunkt der Fahrt zum idealen Zeitfenster; wussten doch die osmanischen Autoritäten schon im Mai 1588 von den spanischen Vorbereitungen zu einer großangelegten Attacke auf die Britischen Inseln:

\footnotetext{
»Dem englischen Agenten [Eduard Burton] wardt angemeldet, das der turckische Keiser in gewisse Erfahrung komen, das der Konigk in Hispanien sich starck in Engelandt zu fallen, weil dan der grose Sultan sich erkennet, seinen Bundtgenossen die Handt zu bitten, wo sie sein begehreten. So es nun die Konigin begehret, wollten ihr die Turcken Hulfe leisten, welches sie itzo gahr wol thuen kuntten, weil der Assam Bassa eine grose Armada bei einander hette ${ }^{65}$."
}

Das Aktionsfeld des westlichen Mittelmeers war im Allgemeinen den Korsaren der osmanischen Satelliten Tunis, Algier, Biserta oder Tripolis vorbehalten. Die nördliche Ägäis und der für die Versorgung Konstantinopels so wichtige Zugang zu den Dardanellen standen unter dem Kommando eines »Hassan Reis« der Flotte des Sultans. Südlich der Insel von Lesbos patrouillierte die Gewässer ein weiterer Verband unter dem Kommando eines »Sancak Bey«. Auch in Alexandria war ein osmanischer Verband von Kriegsschiffen permanent stationiert; ebenso in Suez, von wo aus das Rote Meer kontrolliert werden sollte. Mit der osmanischen Expansion auf die Arabische Halbinsel wurde das Kommando über die in Suez stationierte Flotte aufgewertet und erhielt einen von der militärischen Führung

65 Beschreibung (wie Anm. 12), Bd 2, S. 49. 
über das Mittelmeer unabhängigen Status ${ }^{66}$. Zurzeit von Lubenaus Aufenthalt in Konstantinopel umfasste die osmanische Galeerenflotte 21 im Goldenen Horn stationierte Galeeren, 9 hatten ihre Basis im Hafen von Rhodos, 3 in Chios, 2 auf Zypern, 2 in Alexandria, 2 in Tripolis, 1 in Damietta, 1 in Nauplia (Návplion), 1 in Lesbos und 1 in Cavalla ${ }^{67}$. Insgesamt standen also am Ende des 16. Jahrhunderts zwischen 45 und 50 Galeeren im permanenten Dienst.

Mit der kontinuierlichen Expansion des Osmanischen Reiches begann sich spätestens in der zweiten Hälfte des 16. Jahrhunderts auch die Verletzlichkeit und Fragilität der zur Aufrechterhaltung der Herrschaft nötigen maritimen Versorgungs- und Kommunikationslinien bemerkbar $\mathrm{zu}$ machen ${ }^{68}$. Ein Schutz dieser Handelsrouten zwischen Alexandria und Konstantinopel, zwischen den Häfen des Peloponnes und Gallipoli, zwischen Tripolis, Tunis und Algier und den Dardanellen wurde zu einer Hauptaufgabe der osmanischen Flotte ${ }^{69}$. Ungeachtet der Stärke der Osmanen war die zentrale Handelsroute zwischen Alexandria und Gallipoli bzw. Konstantinopel stets von florentinischen, maltesischen, englischen, katalanischen oder mallorquinischen Korsaren bedroht. Dies bedeutete häufig die Verpflichtung der Handelsschiffe, in Konvois und unter Begleitung von Kriegsschiffen zu segeln. Bis zum Tod Sultans Suleymans I. herrschte der »Beylerbey« von Algier auch über Tunis und Tripolis. Erst in den 1570er Jahren erhielten die Beys von Tunis und Tripolis eine gewisse Eigenständigkeit unter der Oberhoheit des Sultans. 1577 organisierte die Hohe Pforte ihre nordafrikanischen Satelliten in drei Provinzen, jede von einem General-Statthalter (»Beylerbey Pascha«) geführt.

Die von den sogenannten Barbaresken-Staaten aus operierenden KorsarenKapitäne waren trotz ihrer häufig mit Konstantinopel abgestimmten Aktionen kein integraler Bestandteil der osmanischen Flotte. Sie waren damals in einer sogenannten Gilde, der »Taifet-ül-rü'esa« organisiert. Die meisten dieser Kapitäne waren Konvertiten oder von der Türkei nach Nordafrika migrierte Seeleute. Der »Beylerbey« wurde im Allgemeinen aus der Mitte dieser Korsaren gewählt. Nicht wenige stiegen später in hohe und höchste Ämter im Dienst des Sultans auf; einer von ihnen war der von Lubenau beschriebene, bereits erwähnte Uluç 'Alì

66 Traian Stoianovich, L'espace maritime segmentaire de l'empire ottoman. In: Economies méditerranéennes. Equilibres et intercommunications. XIIIe-XIXe siècles. IIe Colloque International d'Histoire (Athènes, septembre 1983), 3 t., Athen 1985-1987, hier t. 1, S. 293-218.

67 Vgl. auch Anderson, Naval Wars (wie Anm. 62), S. 70 f., 75 f. und S. 79f., und den die Korrespondenz der niederländischen Gesandten am Sultanshof auswertenden Groot, The Ottoman Threat (wie Anm. 3), S. 208.

68 Michel Fontenay, L'Empire ottoman et le risqué corsaire au XVIIe siècle. In: Revue d'Histoire moderne et contemporaire, 32 (1985), S. 185-208.

69 Vgl. Groot, The Ottoman Threat (wie Anm. 3), S. 200-203. 
(»Occhiali«), zum Zeitpunkt seines gewaltsamen Todes das Amt eines »Kapudan Pascha (Paşa)« bekleidend.

Um 1600 verfügte Algier über 6 Galeeren und etwa 60 hochbordige Kriegsschiffe, Tunis über 6 Galeeren und 14 große, nur von Segeln angetriebene Kriegsschiffe und Tripoli lediglich über 2 oder 3 Galeeren. Die Präsenz einer starken Garnison von Janitscharen garantierte in den nordafrikanischen Hafenstädten die Autorität des Sultans. Janitscharen waren ebenfalls vonnöten, sollte die Kaperung fremder Kriegsschiffe beabsichtigt werden. Die unter den Korsaren dienenden Kriegsknechte waren im Allgemeinen nicht in der Lage, den gut ausgebildeten und erfahrenen venezianischen, genuesischen, maltesischen oder spanischen Seesoldaten Paroli zu bieten.

Der bereits erwähnte, auf den landgestützten Feldzügen liegende Akzent der osmanischen Militärmaschinerie dokumentiert sich in der Tatsache, dass weder ein Sultan noch ein Großwesir im 16. Jahrhundert Flottenoperationen persönlich begleiteten. Der Aufstieg des obersten Flottenkommandeurs in den erlauchten Zirkel des »Divan« und damit seine Teilnahme an den Beratungen des höchsten Regierungskreises erfolgte erst mit der Reform und Modernisierung der Flotte unter dem neu ernannten »Kapudan Pascha« Khayr al-din (»Barbarossa «) in den 1530er Jahren. Bis Khayr al-din bekleidete der Statthalter von Gallipoli, wo sich auch die bedeutendsten Werften der Osmanen befanden, gleichzeitig das Amt eines Admirals. 1533 wurde Khayr al-din von Suleyman I. zum Admiral der Flotte (»Kapudan-i derya«) ernannt. Damit und eng verbunden mit Khayr al-dins Visionen einer maritimen Dominanz über das zentrale Mittelmeer erfuhr das Amt eine deutliche Bedeutungserweiterung ${ }^{70}$.

$\mathrm{Zu}$ Zeiten Lubenaus gehörte $\mathrm{zu}$ den wichtigsten Aufgaben eines »Kapudan Paschas« die Kontrolle und Patrouille der Haupthandelsrouten von Konstantinopel nach Ägypten via Chios, Rhodos, Castelorizo, Zypern, Alexandretta, Saida, Akkon und Alexandria, und der zu den nordafrikanischen Hafenstädten via Schios, Andros, Negroponte, Monemvasia, Modon, Navarino, Santa Maura, Prevesa und Tripolis bzw. Biserta, Goletta und Tunis. Als Kommandeur über die Flotte und der damit verbundenen Soldaten war der »Kapudan Pascha« ein entscheidendes Bindeglied zwischen dem Sultan bzw. der Führungsspitze in Konstantinopel und den nordafrikanischen Beys. Jedes Jahr unternahm der »Kapudan Pascha« seine Inspektionstour zu den wichtigsten Mittelmeerhäfen des Osmanischen Reichs. Bei der von Lubenau beschriebenen Unternehmung handelte es sich nicht um die ebenfalls jährlich vom osmanischen Großadmiral durchgeführte Tour durch das östliche Mittelmeer zur Eintreibung der ihm in seiner Eigenschaft

70 Vgl. Imber, The Navy (wie Anm. 30). 
als General-Statthalter der Region zustehenden Steuern. Häufig waren die Inspektionsreisen mit Kaperzügen in christliche Gewässer verbunden. 1576 wurde beispielsweise die Küste Nordsiziliens geplündert, 1587 traf es wie so häufig die Küste von Reggio in Kalabrien. Die Erkundungs- und Kapertour des Jahres 1588 an der Lubenau teilnahm - war aus mehreren Gründen außergewöhnlich; sie fand fast am Ende der Saison statt und wagte sich wie selten zuvor tief in feindliche, das heißt spanische Gewässer.

Der osmanische Großadmiral residierte in einem in der Nähe zu den Werften am Goldenen Horn befindlichen Palast. Er war im Allgemeinen gleichzeitig Statthalter-General des griechischen Archipels - eine Funktion die es ihm erlaubte, direkt über Rekrutierung und Ausstattung der Flotten zu entscheiden. $\mathrm{Zu}$ diesem Territorium gehörten die »Sanjaks« von Gallipoli, Negroponte, Lepanto, Karli-eli, Mistra, Rhodos, Lesbos, Kocaeli, Biga, Sughla (die Region von Izmir), Chios, Naxos, Andros und Mahdia (an der tunesischen Küste). Das Hauptquartier der osmanischen Flotte, »Tersâne i 'Âmire «, befand sich damals am Goldenen Horn ${ }^{71}$.

$\mathrm{Zu}$ den vorrangigen Aufgaben eines Flottenkommandeurs gehörte die Garantie der Instandhaltung und Bereitschaft der Kriegsschiffe. Im Anbetracht der limitierten Kommunikationsmittel, besonders im Fall von schwerer See und Sichtbehinderung, war die direkte Einflussnahme des Geschwaderkommandeurs während eines Kampfes oder der Verfolgung eines Gegners nur begrenzt. Befehle und Signale hatten dementsprechend so einfach wie möglich $\mathrm{zu}$ sein. Lubenau erwähnt in diesem Kontext nicht nur die Signalgebung durch Flaggen, sondern auch durch spezielle Pfeifen. Unserem Chronisten war es sicherlich nicht möglich und gestattet, einen tieferen Einblick in die Absichten und Hintergründe der Operationen Hassan Paschas zu erhalten. Zeitweise erscheinen die von dem Königsberger Apotheker beschriebenen Manöver und Routen als wenig sinnreich oder geografischer Unkenntnis geschuldet, wenn etwa die Flotte Mitte Oktober 1588 zunächst die Einfahrt in den Golf von Tunis vermeidet und weiter entlang der tunesischen Küste segelt, um erst zwei Tage später ein Rückkehrmanöver zu unternehmen. Wir kennen die direkten Hintergründe dieses Manövers nicht, wahrscheinlich war es als nautisches Training geplant oder unternommen worden, um Offiziere und Mannschaften mit den jeweiligen Küsten, Wind- und Meeresverhältnissen des Kanals zwischen Nordafrika und Sizilien vertraut zu machen. Eventuell war auch die Jagd auf christliche Schiffe beabsichtigt.

71 Vgl. Wolfgang Müller-Wiener, Zur Geschichte des Tersâne i ’Âmire in Istanbul. In: Türkische Miszellen. Festschrift für Robert Anhegger. Hrsg. von Jean-Louis Bacqué-Grammont, Istanbul 1987, S. 253-273. 
Ebenso scheint die zeitweise gefährlich nahe Fahrt entlang der von zahlreichen Untiefen und Sandbänken geprägten libyschen und ägyptischen Küste beabsichtigt gewesen $\mathrm{zu}$ sein. Hier wurde an Orten geankert, an denen eine Versorgung der Mannschaften nicht zu erwarten war; dabei kam es wahrscheinlich zur Einübung von Landungen an flachen sandigen Küsten: ein Training zur Vorbereitung von Landrazzien bzw. der Flucht vor überlegenen, primär mit Segelschiffen ausgerüsteten Verbänden. Das Profil von Küsten und die Kapazitäten von Häfen spielten entscheidende Rollen bei der Vorbereitung von maritimen Auseinandersetzungen. Küsten konnten zum Flankenschutz dienen, Ankerplätze oder flache Sandstrände zum letzten Fluchtpunkt vor überlegenen Feindverbänden werden. Tatsächlich wird damals über das Anlandziehen der flachkieligen osmanischen und nordafrikanischen Galeeren in Zeiten der Not berichtet.

\section{Reinhold Lubenau, Hassan Pascha "Veneziano« und Deli Mehmet im östlichen Mittelmeer}

Widmen wir uns im Folgenden Lubenaus Bericht und den von ihm beschriebenen Ereignissen. Lubenaus Aufenthalt in Konstantinopel fiel in eine Phase, in der für die osmanische Marine nach dem legendenumrankten Tod des Veteranen von Lepanto und ehemaligen Korsaren Euldj 'Alī - Lubenau nennt ihn »Oczu Ali« ein neues Zeitalter anbrach. Euldj 'Alīs Nachfolger wurde mit dem früheren General-Statthalter von Tunis, Hassan Pascha »Veneziano«, ebenfalls ein Konvertit. Gemäß Lubenaus Ausführungen war Hassan Pascha Sohn eines venezianischen Fischers und langjähriger Schiffsschreiber auf venezianischen Schiffen gewesen, bevor er im Jahr des christlichen Sieges von Lepanto in osmanische Gefangenschaft geriet, dort zum Islam konvertierte und durch Begabung und Skrupellosigkeit eine steile Karriere absolvierte. Im Januar 1588 erhielt Hassan Pascha wegen seiner maritimen Erfahrung einen »ferman« als »Serdar der Ghazis« und war damit oberster Kommandeur und Koordinator der Flotten der nordafrikanischen Satelliten ${ }^{72}$.

Nach seiner Amtseinsetzung rüstete sich der neue osmanische Admiral für eine Inspektionsreise zu sämtlichen wichtigen osmanischen und den nordafrikanischen Satellitenstaaten des Osmanischen Reichs zugehörigen Häfen und Seefestungen. Insgesamt bestand der von ihm zu diesem Zweck zusammengestellte

72 Vgl. Groot, The Ottoman Threat (wie Anm. 3), S. 235. Vgl. dazu auch Malcolm, Agents of Empire (wie Anm. 28), S. 370f. und S. 392. 
Verband aus «24 Galleen und 6 grosen Bastarden « ${ }^{73}$. Dass diese Neuigkeiten Lubenau von einem »englischen Goldschmidt« zugetragen wurden, dokumentiert erneut die besondere Nähe der englischen Kolonie in Konstantinopel zum Sultanshof und den Spitzen der osmanischen Administration. Lubenau hoffte auf dieser Flotte, eine Passage zu dem 1566 von den Osmanen eroberten Chios zu finden; «hiezu konnte mihr der englische Agent, Herr Eduardus Barton, wol verhelfen ${ }^{74}$. Von der Insel Chios plante er, sodann auf venezianischen Schiffen seine Reise nach Venedig anzutreten. Lubenaus Ansinnen wurde von Barton zunächst mit dem Hinweis auf den geheimen Charakter der Befestigungsinspektionen abgeschlagen. Eine positive Wendung kam, als Lubenau in der Residenz des englischen Gesandten einen der englischen Sprache mächtigen osmanischen Kapitän kennenlernte. Dieser Kapitän (»Reis«), »Deli Mehemet genant « ${ }^{75}$, war im Zuge einer Kaperfahrt des Francis Drake aus spanischer Gefangenschaft befreit worden, hatte sich im Folgenden einige Jahre in England aufgehalten und war dann mit der Gesandtschaft des William Harborne (»Guilhelmus Harborn«) 1578 nach Konstantinopel zurückgekehrt. Durch Vermittlung Deli Mehmets erhielt Lubenau - getarnt als des Kapitäns angeblich englischer Diener - von Hassan Pascha die Erlaubnis, an der Unternehmung teilzunehmen ${ }^{76}$. Lubenau wurde ferner mit geschäftlichen Aufträgen betraut:

»Daneben uberantwortet ehr [Eduard Barton] mihr ein gros Paket turckischer Schreiben an alle und jede Bassa, Beglerbegen, Sanigackbegen Chilerdzi, Cadi und alle Verwalter und Richter des gantzen turckischen Reichs, des Inhalts: Wo englische Gefangene gehalten wurden oder derselben einverleibte, die solt man ohn allen Entgeldt bei Verlust derselben Leben, so sie gefangen hilten, los und ledigk lassen ${ }^{77}$.«

Am 24. September 1588 schiffte sich Lubenau auf der Galeere des Deli Mehmet ein, ihm wurde in der Mitte des Schiffs »eine Bank [zugewiesen], darauf ich mein Lageer haben sollte ${ }^{78}$. Am folgenden Tag legte die versammelte Flotte unter musikalischer Begleitung ab. Interessant sind Lubenaus Hinweise, wie sich der Schiffsverband vor allem nach Pfeifen von Signalen formierte; »und alles mit Pfeiffen der silbern Ziflitten, die sie untereinander so genau kenneten, und aufeinander Achtung hatten, wie sie in der Ordnung fahren sollten, gleichsam zu

73 Beschreibung (wie Anm. 12), Bd 2, S. 120.

74 Ebd., S. 121.

75 Ebd., S. 122.

76 Ebd., S. 124.

77 Ebd.

78 Ebd., S. 125. 
einem Angrif «9 ${ }^{79}$ Der Königsberger Chronist gibt einen genauen Einblick in die Formation:

»Erstlich fuhr der General Obriste mit seiner Galleen forne allein [...] stracks hinder im die funf grose Bastarden oder Galleaeen, die prechtigke, gros und mit vielem Volck und Geschütz besetzt in einer Reigen. Diese alle wahren mitt den andern Galleen gleich einem halben Mohn von hinden herumb beschlossen. Auf beiden Seitten wahren von etzlichen Galleen Flugel. Diese im Fortfahren kamen schnel in einer schonen Ordnung und beschlossen von forne die grosen Bastarden ${ }^{80}$.«

Das Goldene Horn wurde unter dem Abfeuern zahlreicher Salutschüsse verlassen. In der Nähe des Landungsstegs vor dem Serail des Sultans wurde kurz geankert und $\mathrm{zu}$ Ehren Murads III. eine besondere Zeremonie abgehalten. Der »Obristen Visir Bassa« erschien auf dem Steg und übergab Hassan Pascha »eine weisse, eine rote und eine blaue [Fahne], alle vol guldener Sternen, der halb Mon in der Mitten « ${ }^{81}$. In den folgenden Tagen ging es mit gutem Wind in westlicher Richtung durch das Marmarameer. Anlässlich eines Zwischenstopps im Hafen der Insel von Marmara sah Lubenau das schwere Los der dort den Marmor brechenden und bearbeitenden christlichen Sklaven ${ }^{82}$.

Bevor man Gallipoli erreichte, wurde auf der asiatischen Seite der Dardanellen eine große Karacke beobachtet;

»und weil es eine venetianische Kraken wahr, wurde sie gefraget, was sie neues von der hispanischen und englischen Armada gehoret, ob sie auch der Malteser, Hispanier und anderer Christen Galleen in Mehr vermercket, was neues zu Venedigk aus dem romischen Reich gehöret wurde, von Maximiliano und dem Konige von Pohlen ${ }^{83}$.«

Kurze Zeit später erreichte die Flotte den Hafen von Gallipoli, wo sie mit Ehrensaluten von der Festung begrüßt wurde. Von militärgeschichtlichem Interesse sind Lubenaus Kommentare zu der Werft und dem Arsenal der Stadt, »darin wahren dieses mahl zehen groser Galleen, und lagen etliche und zwantzigk groser Stuck dabei $\ll^{84}$.

In den folgenden Tagen auf See war Lubenau häufig Gast am Tisch Hassan Paschas und musste sich nicht selten Bekehrungsversuche des Flottenchefs an-

79 Ebd.

80 Ebd., S. 126.

81 Ebd., S. 128.

82 Ebd., S. 131.

83 Ebd., S. $132 \mathrm{f}$.

84 Ebd., S. 134. 
hören. Im Allgemeinen wurde dabei das Motiv der im Vergleich zu den christlichen Ländern ungleich durchlässigeren Hierarchien herausgestrichen:

»[I]ch wehr ein hurtiger, junger Man, der schon viel Lender gesehen, mancherlei Felle erfahren, wuste allerlei Gelegenheit, und konnte den turckischen Soltan wol nutze werden; den bei ihnen wehr kein Ansehen des Herkommens. Ehr wollte mihr balde ein Galleren untergeben und konnte wol konftig an seine Stelle kommen. Ehr wahr auch eines schlechten Herkommens, eines Fischers Sohn von Venedigk und ein geborener Christ, welches sich die turckischen Herren am meisten ruhmen. Den der Ibrahim Bassa, eines Schweinehirts Sohn auf Dalmatia in Croia wahr und hette des Keisers Tochter zur Ehe und zu solchen Ehren komen sie durch Tugent, und wirdt von keinem Adel oder Geschlecht bei ihnen etwas gehalten ${ }^{85} . \ll$

Der deutsche Mediziner blieb dennoch standhaft; andere auf den osmanischen Schiffen segelnde Soldaten und Seeleute blieben es nicht. Lubenau berichtet vom Fall eines Engländers, der »fünf Jahr zuvor, ehe die Englischen die Verbundtnus mit dem Turcken gemachet, in Barbarie wahr gefangen worden und $\mathrm{zu}$ ihrem Glauben sich begeben ${ }^{86}$. Auf einer anderen Galeere fuhr ein zuvor von algerischen Korsaren gefangener Hamburger, der sich, um dem Los eines Rudersklaven zu entgehen, als »Barbier « ausgegeben hatte ${ }^{87}$.

Nach der Rückkehr zur Flotte war Lubenau Zeuge, wie ein Eilbote des Sultans Hassan Pascha darüber informierte, dass sich nach Bekanntwerden des Todes von Uluç 'Alī Verbände der »Maltheser, des Pabsts, der Sizilianer, der Florentiner und Jenueser« zu Plünderungen osmanischer Territorien zusammengefunden hätten $^{88}$. Mit dieser Nachricht war die Anweisung des Sultans verbunden, die Verteidigungsbereitschaft der osmanischen Festungen und Häfen auf den griechischen Inseln, namentlich von Lemnos und Negroponte, $\mathrm{zu}$ inspizieren. Zur Verstärkung der Besatzung dieser Festungen musste ein Teil der Galeeren zurück nach Gallipoli fahren und dort Truppenkontingente an Bord nehmen. Am folgenden Tag - dem 28. September - erreichte die wieder vereinte Flotte die beiden den Durchgang der Dardanellen bewachenden Festungen;

»sobalde nun unsere Galleen der Castel ansiehtigk wurden, lissen sie alle Sigel fallen und durch Andeuttung der Pfeiffen wurden alle Galleen in eine feine Schlachtordnung gebracht, einem halben Mohn gleich. Der General aber mit seiner Galleen hilt in der Mitten und sendet mit einer Frigatten etliche Briefe zu den Castellen, und lis etzliche Stuck Geschutz $\mathrm{ab}^{89}$.«

\footnotetext{
85 Ebd., S. 137.

86 Ebd., S. 139.

87 Ebd., S. 143.

88 Ebd., S. 150.

89 Ebd., S. 151.
} 
Die jeweils auf der europäischen (Kastell von »Sestos«) und asiatischen Küste (Kastell von »Abydos«) befindlichen Kastelle erfüllten damals nicht nur militärische, sondern auch zolltechnische Aufgaben ${ }^{90}$.

Nächste Station der Flotte war die Insel Lemnos (Límnos);

»und nachdem der General mit einer Frigaten zum Castel Briefe gesandt, hatt er seinen Brauch nach ein Stuck abgehen lassen, die vom Castel auch eins, und ist die gantze Armada in einer feinen Ordnung in den Port gefahren und haben die Anker fallen lassen « ${ }^{91}$.

Nach der Ladung größerer Mengen von Proviant (u. a. »Cisern, Bonen, Linsen, Zwibeln, Knoblauch ${ }{ }^{92}$ ) erfolgte die Weiterreise in Richtung von Tenedos, wo die Flotte am 30. September Anker warf. Wenige Stunden später traf die Nachricht ein, dass

»in Macedonia bei Nigroponto die Maltheser mit den Sicilianern, Florentinern und Genuesern mit ihren Galleen eingefallen, und [...] da viel Vihe und Schafe, auch etliche Menschen geraubet $\ll^{93}$.

Hassan Pascha gab umgehend den Befehl, »sich fertigk zu machen, ihnen nachzueilen, odern den Pas zuverlegen ${ }{ }^{94}$. Aus diesem Grund passierte man die Inseln Lesbos (Lésvos), Chios (Khíos), Andros, und Tínos ohne Anlandung. Während dieser Passage behielt die Flotte ihre feste Fahrtordnung:

»Es muste die kleineste Gallee immer vorhero fahren, welche drei Laternen hinder auf der Puppa [Heck] hatte; der volgeten die andern hindenach, jede mit ihren Laternen, welches bei der Nacht im Mehr gahr schon anzusehen wahr, sonderlich, da solch eine Menge der Galleen beieinander ist ${ }^{95} . \ll$

Bevor man sich Negroponte (Évvoia) näherte - es war der 1. Oktober -, wurden »etliche Frigatten ausgesandt, nach der Christen Galeen zu forschen ${ }^{96}$.

Wie schon von den Fregatten gemeldet, wurden im Hafen von Negroponte keine christlichen Kriegsschiffe gefunden. Lubenau beschrieb die beiden den Hafen und die Meerenge zwischen Évvoia und dem Peloponnes bewachenden Festungen als mit außergewöhnlich starken Basteien versehen. Der Königsberger hatte im Folgenden die Gelegenheit, Hassan Pascha auf seinen Inspektionstouren durch die Festungen zu begleiten; »wahren in beiden Festungen etlich Hundert

90 Ebd., S. 154.

91 Ebd., S. 157.

92 Ebd., S. 165.

93 Ebd., S. 168.

94 Ebd.

95 Ebd., S. 173.

96 Ebd., S. 174. 
groser, gegossener Stuck Geschutz, die zum Theil gahr alt « ${ }^{97}$. Viele von ihnen waren im Abendland hergestellt worden ${ }^{98}$. Interessant ist, dass es Lubenau im Folgenden gestattet wurde, Zeichnungen der Festungen anzufertigen. Nach der Inspektionstour befahl Hassan Pascha die Weiterfahrt nach Attica und dem Hafen von Piräus. Nach dort vorgenommener neuer umfangreicher Verproviantierung begab sich Hassan Pascha nach Athen und ließ dort sämtliche Festungskommandanten und höheren osmanischen Offiziere der in der Region stationierten Truppen zur Lagebesprechung zusammenkommen. In der Zwischenzeit wurde Lubenau von drei dort stationierten Janitscharen verhaftet; sie beschlagnahmten seine "grose Schreibtafel, darin [er] viel Sachen abgerissen und viel grichischer Schriften $\mathrm{zu}$ Nicea, Nicomedia und sonsten hin und wieder abgeschrieben hette, und wollten mich als einen Verrehter wegkschleppen ${ }^{99}$. Gegen das Bakschisch von einem Dukaten wurde er wieder auf freien Fuß gesetzt.

Am 5. Oktober verließ die Flotte den Hafen, am nächsten Tag erreichte sie die Hafenstadt Korinthos. Dort räumten auf Befehl von Hassan Pascha weitere hundert Janitscharen die Galeeren, um die Besatzung der über der Hafenstadt befindlichen Bergfestung zu verstärken ${ }^{100}$. Nächste Station war Návplion ${ }^{101}$. Lubenau war von der über der Stadt thronenden Palamidi-Festung beeindruckt. Als einer der wichtigsten osmanischen Militärhäfen war der »schöne Port zwischen Stadt und Lande« gegen Angriffe von der Landseite durch ein »sonderbahres, groses Rundehl am Ufer des Meeres « und eine daran anschließende »bis an das Geburge (reichende) gewaltige starcke Mauer« geschützt:

»Den es hat viel Seheporten in Morea, da keine Festungen bei sindt und konnten der Christen Galleen, [...] da ankomen, und bei Nacht sich uber Landt in diesen Port machen, und die Schiffe so da legen, berauben, wan diese Maur nicht wehre ${ }^{102}$."

Am 7. Oktober legte die Flotte von Návplion ab und segelte in südlicher Richtung zum Kap von Maléa, wo die Galeeren von einem schweren Sturm überrascht wurden. Er zwang den Verband vom Kurs abzuweichen und im Hafen der Insel Mílos Schutz zu suchen. Lubenau beschrieb die Hauptstadt der Insel von lediglich einem »gahr schlechten, viereckichten Castel« verteidigt. Am 8. Oktober wurde ein Verband von sechs spanischen Galeeren gesichtet, doch bevor die »armen Sclaven mit aller Gewaldt« die osmanischen Schiffe aus dem Hafen rudern

97 Ebd., S. 175.

98 Ebd.

99 Ebd., S. 179.

100 Ebd., S. 181.

101 Ebd., S. 186.

102 Ebd., S. 187. 
konnten, "wahren die hispanischen Galleen schon des Weges auf Candia gefahren, das man sie nicht mehr sehen kunte ${ }^{103}$. Mit dem venezianischen Kíthira (»Cerigo«) und seinem »schönen Hafling « (Hafen) erreichte man erstmals christliches Territorium, „und weil die Turcken mit den Venetianern Friede haben, mögen sie wol allhie einlaufen, welches unter ihnen also verglichen ${ }^{104}$. In Kíthira liegt »eine kleine Festung mit Soldaten besetzet, Cithero genandt, leidt aber nicht hardt am Port, sondern etwa im Lande ${ }^{105}$.

\section{In »christlichen« Gewässern}

Von nun an war Vorsicht geboten, segelte man doch in feindlichen Gewässern. Dies erforderte besondere Vorkehrungen, »haben die Turcken uber drey Stunden nicht geschlafen, sondern im Mitternacht den 9. Octobris aufgebrochen ${ }^{106}$. Der günstige Nordwind (»Tramontana«) ermöglichte eine außergewöhnlich schnelle Überfahrt in sizilianische Gewässer. Obwohl eigentlich Tunis Zielhafen war, erachtete Hassan Pascha seinen Verband für stark genug, eine Begegnung mit Kriegsschiffen des Vizekönigreichs Sizilien zu riskieren:

»Den 10. Octobris in gutter Ordnung bei Sicilien komen und lencketen die Turcken mit Fleis nach dem Lande, die sicilianische Galleen heraus zu locken. Wier kamen so nahe nach dem Lande, das wier auch die Festung Saragozam (Syrakus) sehen kunten. Es kamen aber keine heraus $^{107}$."

Das Gleiche wurde vor der Johanniterresidenz Malta versucht:

»Die Turcken naheten sich gegen Maltam, kamen auch so nahe, das die von der Festung, die man gahr wol sehen kunte [...] auf uns geschossen, das die Kugeln nicht weidt von den Galleen fur uns ins Wasser fielen. Die Turcken hetten gerne der Maltheser Galleen herausgelocket gehabt; aber wier wahren ihnen zu starck, kuntten also nichts ertappen ${ }^{108}$."

Hier war die osmanische Führung nicht über den aktuellen Stand der Dinge informiert. Die maltesische Galeerenflotte befand sich damals nicht im Porto Grande der Insel, sondern auf der Rückreise von Genua ${ }^{109}$.

103 Ebd., S. 191.

104 Ebd.

105 Ebd.

106 Ebd., S. 192.

107 Ebd.

108 Ebd.

109 Vgl. Freller, Spies (wie Anm. 11), S. 148. 
Die Fahrt wurde weiter Richtung Tunis fortgesetzt. In der Mitte des Kanals zwischen der nordafrikanischen Küste und der Insel Pantelleria wurde der Hauptmast von Deli Mehmets Schiff mit der »antenna« verlängert, »zu sehen, ob etwa Christengalleen oder Schiffe verhanden wehren «"110. Tatsächlich meldete der »Spion« »zur rechten Handt dreyer Christen Galleen «"111. Umgehend wurde die Verfolgung aufgenommen und die "armen Sclaven musten mechtigk rudern «"12. Die Jagd zog sich allerdings in die Länge und die »zwei fordersten Galeen entgingen uns und kamen gehen Calaris [Cagliari] in den Port, in die gewaltige, schöne Stadt in Sardinia, so itzo Calagir auch Cagler von den Turcken genandt « ${ }^{113}$. Die dritte christliche Galeere - Lubenau nennt nicht das Herkunftsland - wurde fast eingeholt, doch einbrechende Dunkelheit und ein starker Wind verhinderten schließlich ein Kapern. Die Galeere konnte sich in den Golf von Oristano und in die Reichweite der Geschütze der gleichnamigen Festung retten. Die Orientierung entlang der sardischen Küste bereitete Hassan Paschas Verband keine Schwierigkeiten, „den die Turcken, so auf den Galleen, seind mehrentheil Italiener und Renegaten und verleugnete Christen; dieselben wissen alle Porten und Schliche in der gantzen Sehe ${ }^{114}$.

Auch im Folgenden ging die Jagd auf christliche Schiffe - entgegen der ursprünglichen Absicht, den Zielhafen Tunis zu erreichen - weiter. Die vom wiederum aufgesetzten »Spion« in der Nähe der Sardinien nördlich vorgelagerten Insel Asinara erspähten »etliche[n] grose[n] Schiffe« konnten jedoch mangels Wind nicht verfolgt werden. Als der Wind schließlich erneut drehte und aus Süden blies, war für den Moment an eine Fortsetzung der Fahrt nach Tunis nicht zu denken. Hassan Pascha entschied sich für eine nördliche Route mit dem Ziel Marseille,

»nun durften die turckischen Galleen in den Inseln sich nicht aufhalten, und nach dem affricanischen Lande wahr unmöglich zu komen. Derwegen sie sich nohthalben nach dem Marsilianischen Port in Frankreich machen musten, weil die Frantzosen mit den Turcken einen ewigen Frieden aufgerichtet haben ${ }^{115}$.«

Bereits am 13. Oktober erreichte die osmanische Flotte die von gefährlichen Riffen und Untiefen geprägte Zufahrt zum Hafen von Marseille. Einmal mehr zeigte sich die Bedeutung der früher in der christlichen Seefahrt beschäftigten Konvertiten.

110 Beschreibung (wie Anm. 12), Bd 2, S. 193.

111 Ebd.

112 Ebd.

113 Ebd.

114 Ebd., S. 194.

115 Ebd., S. 195. 
Dank ihres Wissens erreichten die Schiffe sicher den Hafen. Nach dem Austausch von Salutschüssen und der Verproviantierung erfolgte die Weiterreise. Den osmanischen Seeleuten und Soldaten - und auch Lubenau - war es nicht gestattet worden, an Land zu gehen. Weiter ging es entlang der südfranzösischen Küste in südwestlicher Richtung. Derweil bereitete man sich an Bord auf Landattacken vor; es "suchten die Turcken ein hauffen Leuttern aus den Galleen herfuhr; die wahren gemachet, das man sie ineinander stecken kunte, und mit eisernen Boltzen fest machen, so langk, als sie woltten, etwas zu ersteigen ${ }^{116}$.

Nachdem Barcelona passiert worden und allmählich der Abend angebrochen war, wurde Kurs auf die Küste genommen. Offenbar war die nun folgende Razzia durch ortskundige Seeleute oder Soldaten gut vorbereitet. Mit Beibooten wurden etwa 300 gut bewaffnete Janitscharen an Land gesetzt. Mit schon vorbereiteten Leitern sah sie Lubenau im Landesinneren verschwinden. Einige andere Gruppen von Soldaten sicherten die Küste, wobei sie auch Herden von in der Nähe weidenden Schafen und andere Tiere erbeuteten. Nach einiger Zeit kehrten die Janitscharen an den Strand zurück. Neben mit geraubten Gegenständen beladenen Eseln führten sie auch »etliche junge Weiber und Megdlin, so wol etliche Menner so gebunden« mit sich ${ }^{117}$. Die Beute und die Gefangenen wurden - mit Ausnahme von Hassan Paschas Flaggschiff - auf die anderen Schiffe verteilt. Noch in der Nacht erfolgte mit gutem Wind die Weiterreise in südlicher Richtung. Auf der Höhe von Cap Nao, nördlich von Alicante, drehte die Flotte in südöstlicher Richtung gen nordafrikanische Küste. Der diesen Abschnitt der Reise behandelnde Teil von Lubenaus Tagebuch dokumentiert, in welch hohem Maß die osmanische Führung über die Beschaffenheit der iberischen Levante-Küste und der dort befindlichen Häfen und Städte im Bilde war. Lubenau berichtete auch über die in den Häfen von Mallorca, Ibiza, Valencia oder Alicante gehandelten Waren: Wein, Salz, Anis, Reis, Öl oder Mandeln ${ }^{118}$.

Bei der Gruppe der Galite-Inseln erreichte die Flotte am 16. Oktober die nordafrikanische Küste. Die Hauptinsel (»Calitam«) war damals nicht nur ein berüchtigtes Versteck von »Sehreubern, die keinem Herren unterthan seindt«, sondern auch bekannt als Koralleninsel ${ }^{119}$. Nach kurzer Rast wurde die Reise in östlicher Richtung entlang des Golfs von Tunis und ab Cap Bon dann mit »guttem Windt « in südlicher Richtung fortgesetzt. Von Pantelleria (»dem Konige aus Hispanien zugehorigk, darinnen eine [...] starcke Festung ${ }{ }^{120}$ ) und Malta hielt man sich

116 Ebd., S. 197.

117 Ebd., S. 198.

118 Ebd.

119 Ebd.

120 Ebd., S. 199. 
diesmal fern. Der Golf von Tunis wurde passiert, um sich in voller Beflaggung und »Schlachtordnung « Hafen und Festung von Biserta zu nähern, »welche an einem Schlundt eines Sehes gelegen, da ein schoner groser Mehrhafen fur grose Schiffe, auch etliche im Hafen lagen, die uns viel Ehrenschus theten ${ }^{121}$. Nächstes Ziel war die Tunis und seinen Hafen bewachende Festung von Goletta (heute Halqel Oued). Nach der osmanischen Besetzung wurde Goletta - gleichsam als islamisches Gegengewicht zum kaum 200 Kilometer entfernt gelegenen spanischen Vizekönigreich Sizilien - weiter ausgebaut. Lubenau berichtet:

»Die Festung Goletta ist itziger Zeit gahr starck und fest mitt gewaltigen Pasteien und Streichwehren, gleichsam einem Stern mitt sechs Spitzen, gebauet; hatt einen gewaltigen, ausgefutterten Graben von Quadersteinen; der mittlere Stock ist noch sonderlich mitt vier Spitzen und sonderlichen Pasteien gantz kunstlich gebauet; hatt fur der Festung den grosen Mehrhafen, da die Schiffe und Galleen einlaufen konnen ${ }^{122 . "}$

Der Königsberger Mediziner schreibt über die Zeremonie des Anlandens:

»Wie wier da furuber kamen, muste der General, ob ehr schon alda lange Zeit Bassa gewesen, gleichwol eine Frigaten mit keiserlichen Briefen an die Festung senden. Die von der Festung stecketen stracks otliche Bandera oder Fahnen aus und thaten einen Schus. Die von den Galeen lissen herrlich nach der Ordnung ihr Geschutz abgehen, nachmals die von der Festung, und fuhr der General mit groser Pracht unter die Festung, und lissen alle Galleen ihre Ancker fallen ${ }^{123}$.«

Der Anteil der christlichen Bevölkerung war damals hoch, »sie war[en] gemäß der Turcken Gewonheit nach gekleidet«. Zusammen mit Deli Mehmet begab sich Lubenau - es war der 18. Oktober - in das nur wenige Kilometer entfernte Tunis; »der fuhret mich herumb, zeiget mihr das Arsinal und allerlei Gelegenheit der Stadt « ${ }^{124}$. Am selben Tag wohnte Lubenau dem Begrüßungsbesuch des Beys von Tunis - Lubenau nennt ihn »Bassa« - auf dem Flaggschiff Hassan Paschas bei. Hassan Pascha und Bey Ibrahim begaben sich dann in dessen Palast in Tunis ${ }^{125}$.

Da sich die osmanische Flotte für mehrere Tage im Hafen von Goletta aufhielt, ergab sich für Lubenau die Gelegenheit, zusammen mit Deli Mehmet einigen im Hafen von Ghar El Melh, dem ehemaligen Porto Farina, vor Anker liegenden englischen Schiffen einen Besuch abzustatten. Die nach wie vor guten Kontakte Deli Mehmets zeigten ihre Wirkung:

121 Ebd., S. 200.

122 Ebd., S. 201.

123 Ebd.

124 Ebd., S. 202.

125 Ebd. 
"Der Kapitein und Schipper des Schifs richteten balde die Malzeit zu, decketen unter einem Sigel einen Tisch, welchen sie zugerichtet hatten und tractireten uns gahr herlich nach ihrer Gelegenheit [...] Unter Essens erzehleten uns die Englischen, welcher gestaldt sie die hispanische Armada geschlagen hatten, und gantz zerstreut ${ }^{126}$."

Die Informationen kamen aus erster Hand:

»Es wehren auch diese Schiffe mit dabei gewesen, und hetten den Hispaniern nachgeiaget, und wehren [...] ins Mare Mediteraneum komen ${ }^{127}$.«

Nach der Rückkehr nach Tunis informierte sich Lubenau intensiver über die maritim ausgerichteten Gesellschaften der sogenannten Barbaresken-Staaten; die tunesischen Korsaren begrenzten damals ihre Fanggründe nicht nur auf die Gewässer von »Siciliam, Sardiniam oder Corsicam «"128, sondern hatten sie »auf den grosen Oceanum (Atlantik)« ausgeweitet: »Wartten auf die indianische Kraken, dorfen woll Schiffe vor Lisbon aus dem Port wegknehmen ${ }^{129}$. Marine, Werften und Zulieferer hingen über den Handelsverkehr hinaus vor allem vom Korsarenwesen ab: »Davon werden die Tunisischen Baschen, der Konigk von Algeir und Fessa so reich, welches alles vom Raum herkomet ${ }^{130} \cdot$ « Mit in Tunis an Bord genommener Fracht und Passagieren - darunter auch Mitglieder des Harems des neuen Beys von Tunis - erfolgte schließlich am 20. Oktober die Abreise der Flotte. Hassan Pascha befahl einen östlichen Kurs. Seine Flotte segelte ohne große Vorkommnisse entlang der nordafrikanischen Küste bis nach Tripolis; eine Stadt mit »einem schonen, grosen und weitten Port, und neben dem Port ein groses Castel mit Pastein und starcken Thurmen wol verwahret ${ }^{131}$. Auch die Beys von Tripolis zogen einen großen Teil ihres Einkommens aus dem Korsarenwesen und der Piraterie. Lubenau beobachtete, inwiefern sich »allhie viel Seherauber aufhalten, die Turcke schutzet, dieweil sie im von allem geraubten Gutt das dritte Theil geben «132. Neu ernannter Pascha von Tripolis war der von Lubenaus Bekannten Michael Heberer als ungemein grausam und skrupellos beschriebene Muhammed, ein von der Insel Pantelleria stammender Konvertit ${ }^{133}$.

126 Ebd., S. 203.

127 Ebd., S. 204.

128 Ebd.

129 Ebd.

130 Ebd.

131 Ebd., S. 208.

132 Ebd.

133 Vgl. Heberer, Aegyptiaca Servitus (wie Anm. 8), S. 301f.; Freller, The life (wie Anm. 11), S. 96. 
Die folgende Weiterfahrt der osmanischen Flotte entlang der Küste der Syrte war aufgrund der Untiefen und Sandbänke nicht ohne Gefahren. Der weitere Kurs führte nach Alexandria, das die Flotte am 25. Oktober erreichte. Dort verließ Lubenau die Flotte, um über Zypern, Rhodos, Kreta, Korfu, Dubrovnik und Venedig seine Heimreise anzutreten.

\section{Die Bedeutung der Aufzeichnungen Lubenaus}

Die Beobachtungen des Königsberger Apothekers wie auch jene seiner oben genannten unmittelbaren Zeitgenossen bzw. Mitreisenden dokumentieren eine außergewöhnliche Flexibilität osmanischer Führung; gerade der Umgang mit den nordafrikanischen Fürstentümern und die Integration einer Vielzahl von Konvertiten in die Militärmaschinerie sind dafür gute Beispiele. Sie zeigen jedoch auch durch technische Faktoren und Defizite bestimmte Grenzen der Expansion und militärischen Operationsfähigkeit. Diese Grenzen wiederum demonstrieren, inwiefern sich die in der Epoche der Schlacht von Lepanto ihren Höhepunkt erreichende okzidentale »Türkenfurcht« häufig aus Übertreibungen speiste. Wenn auch durch das Fehlen flankierender landgestützter Operationen von einer nachhaltigen militärischen Bedrohung der christlichen Anrainer des westlichen Mittelmeerraums nach den osmanischen Niederlagen vor Malta und bei Lepanto sowie der erfolgreichen Niederschlagung des Morisken-Aufstandes in Südostspanien nicht mehr die Rede sein konnte, bestand für die Küstenregionen weiterhin die Gefahr, dass osmanisch-nordafrikanische Verbände sie angriffen. Lubenaus Aufzeichnungen geben Zeugnis eines dieser Angriffe, die häufig mit der Versklavung der einheimischen Bevölkerung verbundenen waren. Der Reisende aus Königsberg dokumentiert ferner, inwiefern diese Operationen - gerade mit Blick auf Frankreich - massiv von konfessions- und kulturübergreifenden flexiblen Allianzen profitierten; die hier diskutierte Quelle wird damit gleichsam zum Spiegel der »großen « Geschichte im Kleinen ${ }^{134}$.

Langfristig sollten die Marginalisierung des Mittelmeerraums und die Unfähigkeit des Osmanischen Reichs im globalen Wettlauf um Kolonien, neue Absatzmärkte und technische Innovation mit England oder Frankreich mitzuhalten, zum Niedergang beitragen. Dieser komplexe Prozess konnte natürlich nicht von

134 Zum Kontext dieser flexiblen Allianzen im Mittelmeerraum vgl. kürzlich Nikolas Jaspers und Sebastian Kolditz, Seeraub im Mittelmeerraum. Bemerkungen und Perspektiven. In: Seeraub im Mittelmeerraum. Piraterie, Korsarentum und maritime Gewalt von der Antike bis zur Neuzeit. Hrsg. von Nikolas Jaspert und Sebastian Kolditz, Paderborn [u.a.] 2013 (= Mittelmeerstudien, 3), S. 11-37. 
Reinhold Lubenau am Ende des 16. Jahrhunderts erfasst werden, seine hellsichtigen Kommentare über die negativen Auswirkungen des Atlantikhandels auf die Städte der Levante und über die Abhängigkeit der osmanischen Marine von frischer Zufuhr okzidentaler Spezialisten und Techniker geben jedoch wichtige Hinweise auf wesentliche Momente struktureller Defizite. Gleichzeitig erhalten wir durch seinen Bericht wertvolle Einblicke in die - jenseits des offiziellen Bildes eines strikten christlich-islamischen Antagonismus - damals bestehenden Möglichkeiten sogenannter Arbeitsmigration d.h. der Abwanderung christlicher Spezialisten und Söldner nach Nordafrika und in das Osmanische Reich.

Neben der oben skizzierten »Türkenfurcht« war daher auch das Phänomen einer »Türkenhoffnung « zu beobachten ${ }^{135}$. Dieses hatte nicht nur für den Mittelmeerraum, sondern auch für Zentraleuropa seine Relevanz. Lubenau berichtet auf seinen Mittelmeerreisen über verschiedene, aus deutschen Regionen stammende Personen, welche die sich durch die osmanische Marine oder das osmanische Heer bietenden Beschäftigungs- und Aufstiegmöglichkeiten wahrnahmen. Damit einher gingen nicht nur ein auf militärisches, technisches Fachwissen beschränkter Wissensaustausch, sondern interkulturelle Transferprozesse verschiedenster Natur.

135 Neben den oben erwähnten Abhandlungen von Carl Göllner - Göllner, Turcica (wie Anm. 3) - vgl. auch Winfried Schulze, Reich und Türkengefahr im späten 16. Jahrhundert. Studien zu den politischen und gesellschaftlichen Auswirkungen einer äußeren Bedrohung, München 1978, vgl. bes. S. 155-160; Karl Vocelka, Das Türkenbild des christlichen Abendlandes in der frühen Neuzeit. In: Österreich und die Osmanen. Prinz Eugen und seine Zeit. Hrsg. von Erich Zöllner und Karl Gutkas, Wien 1988 (= Schriften des Instituts für Österreichkunde, 51/52), S. 20-31; Thomas Freller, The Epitome of Europe. Das Bild Maltas und des Ordensstaats der Johanniter in der Reiseliteratur der Frühen Neuzeit, 2 Bde, Frankfurt a.M. 2001 (= Mainzer Studien zur Neueren Geschichte, 8), hier speziell das Kapitel »Türkenkampf« und »Türkenfurcht«. Realität und Fiktion, S. 131-160; und Robert H. Schwoebel, The Shadow of the Crescent. The Renaissance Image of the Turk, New York 1967. 